\title{
Sensitivity Analysis and Optimization Using Energy Finite Element and Boundary Element Methods
}

\author{
Jun Dong* and Kyung K. Choi \\ University of Iowa, Iowa City, Iowa 52242 \\ and \\ Nickolas Vlahopoulos,, Aimin Wang,,$\underline{\underline{\S}}$ and Weiguo Zhang $\mathbb{I}$ \\ University of Michigan, Ann Arbor, Michigan 48109 \\ DOI: $\underline{10.2514 / 1.20811}$
}

\begin{abstract}
This paper presents a continuum-based design sensitivity analysis and optimization of high-frequency radiation problems using the energy finite element method and energy boundary element method. The noise radiated from the vibrating structure at a high-frequency range is obtained through a sequential procedure. The structural energy finite element method calculates structural energy distribution, which is then used as the boundary condition for the energy boundary element method to calculate the energy density at a far-field observation point. For design sensitivity analysis, the direct differentiation method calculates the sensitivity of the exterior noise through the sensitivity of the structural energy density obtained from the energy finite element method. The adjoint variable method calculates the adjoint load from an acoustic energy boundary element method reanalysis, and the adjoint response is obtained from a structural energy finite element method reanalysis. The sensitivity information is obtained by carrying out numerical integration only on the structural finite element part. The proposed design sensitivity analysis approach has been applied in the design of automotive and naval structures to search for the best material layout to achieve the lowest noise level at high frequency.
\end{abstract}

\section{Nomenclature}

$a_{u}(\bullet, \bullet)=$ structural energy bilinear form

$b_{u}(\bullet, \bullet)=$ structural-structural coupling term

$c_{g} \quad=$ group speed

$c_{g B} \quad=$ structural bending group speed

$c_{0} \quad=$ acoustic wave speed

$e \quad=$ energy density

$e^{\prime} \quad=$ partial derivative of energy density

$\boldsymbol{F}_{\text {adj }} \quad=$ structural adjoint load

$f \quad=$ frequency

$G\left(x, x_{0}\right)=$ Green's function for time-averaged energy density

$\boldsymbol{H}=$ stiffness matrix of EBEM

$H\left(x, x_{0}\right)=$ Green's function for time-averaged energy intensity

$h=$ thickness of plate

$h(\bullet) \quad=\quad$ linear integral form in terms of acoustic energy strength

$\boldsymbol{I}=$ energy intensity

$\boldsymbol{J}_{\mathrm{ss}} \quad=$ structural-structural coupling matrix

$\boldsymbol{K}_{s} \quad=$ structural stiffness matrix

$k=$ acoustic wave number

$k_{s B} \quad=$ structural bending wave number

$\ell_{u}(\bullet)=$ structural load linear form

Received 30 October 2005; revision received 26 January 2007; accepted for publication 2 February 2007. Copyright $@ 2007$ by the Authors. Published by the American Institute of Aeronautics and Astronautics, Inc., with permission. Copies of this paper may be made for personal or internal use, on condition that the copier pay the $\$ 10.00$ per-copy fee to the Copyright Clearance Center, Inc., 222 Rosewood Drive, Danvers, MA 01923; include the code 0001-1452/07 \$10.00 in correspondence with the CCC.

${ }^{*}$ Graduate Research Assistant, Department of Mechanical and Industrial Engineering; currently Research and Development Engineer, CAE Division, LMS International; jun.dong@1mscae.com.

${ }^{\dagger}$ Roy J. Carver Professor, Department of Mechanical and Industrial Engineering; kkchoi@ccad.uiowa.edu. Associate Fellow AIAA (Corresponding Author).

${ }^{\ddagger}$ Professor, Department of Naval Architecture and Marine Engineering; nickvl@engin.umich.edu.

${ }^{\S}$ Research Associate, Department of Naval Architecture and Marine Engineering; wangam@engin.umich.edu.

TGaduate Research Assistant, Department of Naval Architecture and Marine Engineering; zhangwg@engin.umich.edu.

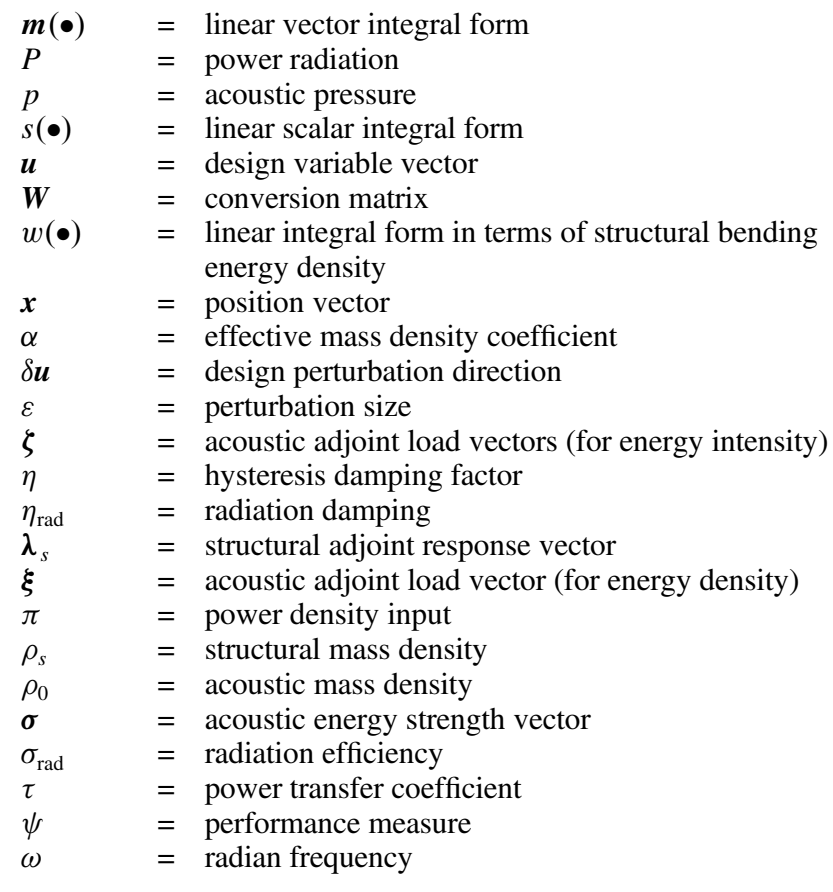

\section{Introduction}

$\mathbf{T}$

HE objective of this paper is to present an analytical approach for the design sensitivity analysis (DSA) of structure-induced sound and vibration at a high-frequency range to design structural systems with desirable acoustic performance while minimizing the mass of the structure. To this end, the energy finite element method (EFEM) and energy boundary element method (EBEM) are used to predict the noise radiated from a vibrating structure, and continuumbased DSA is developed for a sequential EFEM-EBEM procedure for parametric design variables, such as the thickness and material property of a structural plate to evaluate design sensitivity information at the current design and study the potential design change for an optimum design. Both the direct differentiation and adjoint variable methods (AVM) are developed for DSA. 
The reason that the EFEM and EBEM are used for high-frequency structural-acoustic analysis over the traditional finite element method (FEM) [1-3] and boundary element method (BEM) [4-6] is because that the FEM and BEM are proved to be only suitable for structural-acoustic problems at low-frequency range. Because the structural or acoustic domain is discretized in finite elements or boundary elements for FEM or BEM, the element size should be small enough to accurately capture the wave propagation through the structural or acoustic domain. Atalla and Bernhard [7] presented a comprehensive comparison of FEM and BEM in low-frequency structural-acoustic analysis, and they recommended that at least six linear elements or three quadratic elements be required per wavelength for an accurate analysis. From their research, it is commonly agreed that FEM and BEM would only be suitable for a given problem with a maximum frequency of $200 \mathrm{~Hz}$.

Statistical energy analysis (SEA) is an alternative to analyze the response of vibrational structures [ $\underline{8}-10]$ at high frequency. According to SEA, the structural system is divided into subsystems with similar energy modes and the power balance equation is solved to obtain the lumped energy carried by each subsystem. A major drawback for SEA is that the variation of energy response cannot be predicted and design variables do not appear in the governing equation of SEA, so it is not a preferred analysis tool for DSA and optimization.

The EFEM is an application of finite element techniques to the power balance equation to obtain a localized time- and spaceaveraged energy density solution [11-20]. Because energy conservation is imposed locally in power flow analysis, it is possible to represent the structural geometry in detail, which is critical for DSA and optimization. The response variable (energy density) is not continuous across structural junctions and structural-acoustic interfaces in EFEM. To assemble different structural components (rod, beam, membrane, plate, etc.) and to place an acoustic medium inside the built-up structure, a coupling matrix method is developed $[16,17]$ in which the structural-structural and structural-acoustic power transfer coefficients $[20,21]$ are used to consider the power flow conservation between the coupled structural components and structural-acoustic interfaces. The effect of fluid loading on structural vibration at a high frequency is investigated by Zhang et al. [22] and as a result, the power flow governing the differential equation is modified to consider the fluid effect and the application of EFEM is extended to the vibrating structure immersed in dense fluid, that is, naval engineering.

The EFEM has been successfully applied to various engineering problems. Vlahopoulos et al. [23,24] validated EFEM by applying it to a complex ship structure and comparing the EFEM results to SEA and very refined FEM results. Wang [25] used a degenerated version of EFEM to analyze the cabin of a heavy-duty truck.

Because the EFEM requires the discretization of the 3-D acoustic domain, it is not a favorite choice for radiation analysis where the acoustic domain is infinite. For the high-frequency radiation problem, Wang et al. [26] developed an energy boundary element formulation using an acoustic energy corollary. The boundary condition of the EBEM, which is energy intensity on the structural surface, can be obtained either from a structural energy finite element analysis, or by being measured through experiments. This method is applied in the analysis of the radiation of an undersea ship in water [26]. For an analysis of structure borne noise, EFEM and EBEM can be used in a sequential procedure: the structural EFEM solves the structural energy density and the power radiation from the structure, which is then used as a boundary condition of EBEM to compute the radiated far-field noise.

In contrast to significant research efforts in DSA of low-frequency structural-acoustic problems using FEM and BEM [27-38], DSA of high-frequency structural-acoustic problems has not been carried out until Kim et al. [39] proposed a design sensitivity formulation for structural systems (without acoustic coupling) using EFEM. However, in [39], the structural-acoustic relationship was not addressed until Choi et al. [40,41] developed detailed derivations of the parametric design sensitivity formulation for the structuralacoustic coupled problem.
Using the DSA method developed by Choi et al. for EFEM $[40,41]$, the high-frequency radiation noise calculated by a sequential $\overline{E F E M}-E B E M$ procedure can be obtained using either the direct differentiation or adjoint variable method. The direct differentiation method calculates the sensitivity of the radiation noise by differentiating the governing variational equation of EFEM with respect to the design variables. The adjoint variable method calculates the adjoint load from an acoustic EBEM reanalysis, and the adjoint response is obtained from a structural EFEM reanalysis. The sensitivity information is obtained by carrying out numerical integration only on the structural FE part. Both methods present excellent numerical accuracy compared with the finite difference method (FDM).

\section{Theoretical Background}

\section{A. Energy Finite Element Method}

The governing equation of EFEM is derived for the time- and space-averaged energy density $e$ as [11-20]

$$
-\frac{c_{g}^{2}}{\eta \omega} \nabla^{2} e+\eta \omega e=\pi
$$

The first term on the left side represents the transmitted power from the local system to the outside and adjacent systems, whereas the second term on the left side represents the time- and space-averaged dissipated power inside the local system. Equation (1) describes energy conservation in a local system: energy flow into the structure is equal to the energy dissipation from the structure plus the energy flow across the boundaries. This formula can be applied to both structural and acoustic wave motions in similar forms. For a complex structural-acoustic system, $e$ is not continuous on the geometric and material discontinuities. However, the energy flow remains continuous on the structural-structural junctions and structuralacoustic interfaces.

If the interior acoustic domain is ignored in the radiation analysis, a variational equation can be derived for the structural part from governing Eq. (1) using the energy conservation law as

$$
a_{u}\left(\boldsymbol{e}_{s}, \overline{\boldsymbol{e}}_{s}\right)+b_{u}\left(\boldsymbol{e}_{s}, \overline{\boldsymbol{e}}_{s}\right)=\ell_{u}\left(\overline{\boldsymbol{e}}_{s}\right), \quad \forall \overline{\boldsymbol{e}}_{s} \in Z_{s}
$$

In Eq. (2), the subscript $s$ is used to denote the energy density on the structural domain, $Z_{s}$ is the kinematically admissible energy space, and the subscript $\boldsymbol{u}$ indicates the design dependency of the energy terms. Among the energy terms, $a_{\boldsymbol{u}}(\bullet, \bullet)$ is the energy bilinear form, $\ell_{u}(\bullet)$ is the load linear form, and $b_{u}(\bullet, \bullet)$ is the coupling term between structural subdomains. The coupling term is difficult to express in continuum form, but a structural-structural coupling matrix $\boldsymbol{J}_{\mathrm{ss}}$ can be constructed to express $b_{\boldsymbol{u}}(\bullet, \bullet)$ in discrete form as

$$
b_{\boldsymbol{u}}\left(\boldsymbol{e}_{s}, \overline{\boldsymbol{e}}_{s}\right)=\overline{\boldsymbol{E}}_{s}^{T} \boldsymbol{J}_{\mathrm{ss}} \boldsymbol{E}_{s}
$$

Discretization of the variational Eq. (2) leads to the system equation of EFEM as

$$
\left(\boldsymbol{K}_{s}+\boldsymbol{J}_{\mathrm{ss}}\right) \boldsymbol{E}_{s}=\boldsymbol{F}_{s}
$$

The solution of Eq. (4) yields $\boldsymbol{E}_{s}$, the nodal energy density vector on the structure. Because the coupling matrices $\boldsymbol{J}_{\mathrm{ss}}$ are constructed from structural-structural power transfer coefficients, which are nonsymmetric due to the power transfer natures between structural components, the system equation is unsymmetric. The expressions of the energy bilinear form $a_{\boldsymbol{u}}(\bullet, \bullet)$, coupling term $b_{\boldsymbol{u}}(\bullet, \bullet)$, the load linear form $\ell_{u}(\bullet)$, and the expressions of the structural stiffness matrices $\boldsymbol{K}_{s}$ and structural-structural coupling matrix $\boldsymbol{J}_{\mathrm{ss}}$ can be found in Choi et al. [40,41].

\section{B. Energy Boundary Element Method}

The basic integral equation of the EBEM formulation is derived from the acoustic energy corollary [26]. Consider an acoustic medium with volume $V$ which encloses some acoustic energy sources, the area surrounding the volume $V$ is $S$ as shown in Fig. $\underline{1}$, 


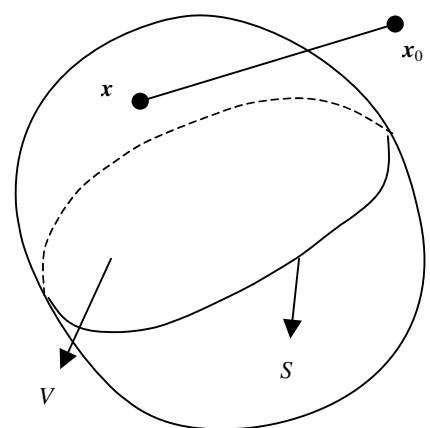

Fig. 1 Arbitrary volume $V$ bounded by surface $S$.

where $\boldsymbol{I}$ is the acoustic energy intensity on the boundary $S$ in the acoustic medium. The total time-averaged acoustic power $P$ radiated by the energy sources can be obtained by the following integral equation:

$$
P=\int_{S} \boldsymbol{I} \cdot \boldsymbol{n} \mathrm{d} S
$$

where $\boldsymbol{n}$ is the unit normal vector pointing out of the volume.

Consider a spherical energy source with strength $\sigma$ located at a field point $\boldsymbol{x}$. The time-averaged energy density and the timeaveraged energy intensity at an arbitrary field point $\boldsymbol{x}_{0}$ due to this acoustic energy source can be calculated by

$$
\begin{gathered}
e\left(\boldsymbol{x}_{0}\right)=G\left(\boldsymbol{x}, \boldsymbol{x}_{0}\right) \sigma \\
\boldsymbol{I}\left(\boldsymbol{x}_{0}\right)=H\left(\boldsymbol{x}, \boldsymbol{x}_{0}\right) \boldsymbol{u}\left(\boldsymbol{x}, \boldsymbol{x}_{0}\right) \sigma
\end{gathered}
$$

where $\boldsymbol{u}\left(\boldsymbol{x}, \boldsymbol{x}_{0}\right)$, is the unit normal vector pointing from point $\boldsymbol{x}$ to $\boldsymbol{x}_{0}$; $G\left(\boldsymbol{x}, \boldsymbol{x}_{0}\right)$ and $H\left(\boldsymbol{x}, \boldsymbol{x}_{0}\right)$ are the corresponding Green's functions for the time-averaged acoustic energy density and the time-averaged acoustic energy intensity in the free field, respectively, and

$$
\begin{gathered}
G\left(\boldsymbol{x}, \boldsymbol{x}_{0}\right)=\frac{\rho_{0}}{64 \pi^{2} r^{4}\left(\boldsymbol{x}, \boldsymbol{x}_{0}\right)}+\frac{k^{2} \rho_{0}}{32 \pi^{2} r^{2}\left(\boldsymbol{x}, \boldsymbol{x}_{0}\right)} \\
H\left(\boldsymbol{x}, \boldsymbol{x}_{0}\right)=\frac{k^{2} \rho_{0} c_{0}}{32 \pi^{2} r^{2}\left(\boldsymbol{x}, \boldsymbol{x}_{0}\right)}
\end{gathered}
$$

In Eqs. (8) and (9), $\rho_{0}$ is the acoustic mass density, and $r\left(\boldsymbol{x}, \boldsymbol{x}_{0}\right)$ is the distance from the acoustic energy source to the field point.

The above equation can be extended from a single energy source to a group of incoherent acoustic energy sources, which then constitutes the governing equation of EBEM. Consider an arbitrary structure that is bounded by a surface $S$ in an infinite acoustic medium, with the surface divided into quadrilateral and triangular elements and each element acts as an incoherent acoustic energy source with strength $\sigma_{j}$ $(j=1,2,3, \ldots, N B E)$. Then the time-averaged acoustic energy density and acoustic energy intensity at a general field point $x_{0}$ in the space outside of the structure can be calculated by the superposition of Eqs. (8) and (9) for each energy source as

$$
\begin{gathered}
e\left(\boldsymbol{x}_{0}\right)=\sum_{j=1}^{N B E} \int_{S_{j}} G\left(\boldsymbol{y}, \boldsymbol{x}_{0}\right) \frac{\sigma_{j}}{A_{j}} \mathrm{~d} S=\sum_{j=1}^{N B E} \frac{\sigma_{j}}{A_{j}} \int_{S_{j}} G\left(\boldsymbol{y}, \boldsymbol{x}_{0}\right) \mathrm{d} S \\
\boldsymbol{I}\left(\boldsymbol{x}_{0}\right)=\sum_{j=1}^{N B E} \int_{S_{j}} H\left(\boldsymbol{y}, \boldsymbol{x}_{0}\right) \boldsymbol{u}\left(\boldsymbol{y}, \boldsymbol{x}_{0}\right) \frac{\sigma_{j}}{A_{j}} \mathrm{~d} S \\
=\sum_{j=1}^{N B E} \frac{\sigma_{j}}{A_{j}} \int_{S_{j}} H\left(\boldsymbol{y}, \boldsymbol{x}_{0}\right) \boldsymbol{u}\left(\boldsymbol{y}, \boldsymbol{x}_{0}\right) \mathrm{d} S
\end{gathered}
$$

In Eqs. (10) and (11), $N B E$ is the number of elements on the surface, $A_{j}$ is the area of the $j$ th element, $\boldsymbol{y}$ is an arbitrary point on the $j$ th element, $\boldsymbol{u}\left(\boldsymbol{y}, \boldsymbol{x}_{0}\right)$ is the unit normal vector pointing from point $\boldsymbol{y}$ to the field point $\boldsymbol{x}_{0}$. Because the energy strength $\sigma_{j}$ is supposed to be constant on a given element, it can be brought outside of the integral with the area $A_{j}$. The above equations can be written in continuum form as

$$
\begin{gathered}
e\left(x_{0}\right)=s\left(x_{0} ; \boldsymbol{\sigma}\right) \\
\boldsymbol{I}\left(\boldsymbol{x}_{0}\right)=\boldsymbol{m}\left(\boldsymbol{x}_{0} ; \boldsymbol{\sigma}\right)
\end{gathered}
$$

where $s(\bullet)$ and $\boldsymbol{m}(\bullet)$ are linear scalar and vector integral forms in terms of the acoustic energy strength, respectively.

If the field point $\boldsymbol{x}_{0}$ is located on the surface, then Eq. (11) can be used to calculate the acoustic energy intensity of each element on the surface. For example, if the field point $\boldsymbol{x}_{0}$ is evaluated at an arbitrary point $z$ inside the $i$ th element, the energy intensity at this point $z$ can be calculated by substituting $x_{0}$ in Eq. (11) by $z$ as

$$
\boldsymbol{I}(\boldsymbol{z})=\sum_{j=1}^{N B E} \frac{\sigma_{j}}{A_{j}} \int_{S_{j}} H(\boldsymbol{y}, \boldsymbol{z}) \boldsymbol{u}(\boldsymbol{y}, \boldsymbol{z}) \mathrm{d} S
$$

Once the energy intensity of an acoustic energy source $\boldsymbol{I}_{i}$ of the $i$ th element on the surface is known, the acoustic power radiated from this energy source into the acoustic medium can be obtained from Eq. (5) as

$$
\int_{S_{i}} \boldsymbol{I}_{i} \cdot \boldsymbol{n}_{i} \mathrm{~d} S=P_{i}, \quad(i=1,2, \ldots, N B E)
$$

where $P_{i}$ is the acoustic power radiated by the $i$ th element. Substitution of Eq. (14) into Eq. (15) leads to

$$
\begin{gathered}
\int_{S_{i}}\left[\sum_{j=1}^{N B E} \frac{\sigma_{j}}{A_{j}} \int_{S_{j}} H(\boldsymbol{y}, \boldsymbol{z}) \boldsymbol{u}(\boldsymbol{y}, \boldsymbol{z}) \mathrm{d} S\right] \cdot \boldsymbol{n}_{i} \mathrm{~d} S=P_{i} \\
(i=1,2, \ldots, N B E)
\end{gathered}
$$

If we write the acoustic power $P$ radiated from each element and the acoustic energy strength $\sigma$ of each element in vector forms as

$$
\begin{aligned}
& \boldsymbol{P}=\left\{\begin{array}{llll}
P_{1} & P_{2} & \cdots & P_{N B E}
\end{array}\right\}^{T} \\
& \boldsymbol{\sigma}=\left\{\begin{array}{llll}
\sigma_{1} & \sigma_{2} & \cdots & \sigma_{N B E}
\end{array}\right\}^{T}
\end{aligned}
$$

then Eq. (16) can be rewritten in matrix form as

$$
H \sigma=P
$$

where matrix $\boldsymbol{H}$ is defined as the stiffness matrix of EBEM, and its component is derived from Eq. (16) as

$$
H_{i j}=\int_{S_{i}}\left[\int_{S_{j}} \frac{H(\boldsymbol{y}, \boldsymbol{z}) \boldsymbol{u}(\boldsymbol{y}, \boldsymbol{z})}{A_{j}} \mathrm{~d} S\right] \cdot \boldsymbol{n}_{i} \mathrm{~d} S
$$

The numerical evaluation of $H_{i j}$ can be obtained by applying the Gauss quadrature in Eq. (20). However, the diagonal terms of matrix $\boldsymbol{H}$ when $i=j$ cannot be computed by the above equation because there is a singularity occurring in the Green's function in Eq. (9) when $r\left(\boldsymbol{x}, \boldsymbol{x}_{0}\right)=0$. To overcome this singularity, Wang [26] pointed out that for a spherical energy source with strength $\sigma_{i}$, the total power radiated by the energy source could be calculated by

$$
\Pi=\frac{k^{2} \rho_{0} c_{0}}{8 \pi} \sigma_{i}
$$

The power associated with the acoustic energy source distributed over an element through one side of the element surface is one-half of the total power П. Therefore, Eq. (15) can be rewritten as

$$
\int_{S_{i}} \boldsymbol{I}_{i} \cdot \boldsymbol{n}_{i} \mathrm{~d} S=\frac{k^{2} \rho_{0} c_{0}}{16 \pi} \sigma_{i}
$$


As a result, the diagonal term of the stiffness matrix in Eq. (19) can be calculated by

$$
H_{i i}=\frac{k^{2} \rho_{0} c_{0}}{16 \pi}
$$

To solve the acoustic energy strength in Eq. (19), the acoustic power radiated from each element needs to be identified from EFEM analysis. As discussed before, the solution of the EFEM will yield the energy density on the structural domain. Then, the acoustic power radiated from the structure to the acoustic medium can be obtained by

$$
P_{i}=\int_{S_{i}} \eta_{\mathrm{rad}} \omega e_{s B} h \mathrm{~d} S
$$

where $e_{s B}$ is the structural bending energy density, $\eta_{\mathrm{rad}}$ is the radiation damping, which is a function of the structural mass density $\rho_{s}$, panel thickness $h$ and geometry and the acoustic wave number $k$, and can be calculated by [42]

$$
\eta_{\mathrm{rad}}=\frac{\rho_{0}}{\rho_{s}} \frac{1}{\alpha k h} \sigma_{\mathrm{rad}}
$$

The parameter $\alpha$ is added to the structural mass density $\rho_{s}$ to represent the effective mass density, which can be calculated as [22]

$$
\alpha= \begin{cases}1 & f>f_{c} \\ 1+\frac{\rho_{0}}{\rho_{s} h \sqrt{k_{s B}^{2}-k^{2}}}, & f>f_{c}\end{cases}
$$

where $f_{c}$ is defined as the coincidence frequency, at which the structural bending wave number $k_{s B}$ coincides with the acoustic wave number $k$. In Eq. (25), $\sigma_{\text {rad }}$ is the radiation efficiency, which quantifies the interaction between the structural bending wave and the acoustic wave, and can be calculated by [43]

$$
\sigma_{\mathrm{rad}}= \begin{cases}\frac{a+b}{\pi \mu k a b\left(\mu^{2}-1\right)^{\frac{1}{2}}}\left\{\ln \left(\frac{\mu+1}{\mu-1}\right)+\frac{2 \mu}{\mu^{2}-1}\right\} & f<f_{c} \\ \sqrt{\frac{a}{\lambda_{c}}}\left(1+\frac{1}{\sqrt{r}}\right) & f \approx f_{c} \\ \left(1-\frac{f_{c}}{f}\right)^{-\frac{1}{2}} & f>f_{c}\end{cases}
$$

where $r=a / b$ is the ratio between the characteristic length $a$ and $b$ of the plate, $\mu=k_{s B} / k$ is the wave number ratio, and $\lambda_{c}=c / f_{c}$ is the acoustic wavelength at the coincidence frequency.

Because of the differences of the acoustic wave numbers and the effective structural mass density of the structural plate in light fluid (air) and dense fluid (water), the radiation damping, which represents the capacity of the structural plates to radiate acoustic energy, will be different in dense fluid compared to that in light fluid. For example, for an aluminum plate with the dimension of $1 \times 1 \times 0.001 \mathrm{~m}^{3}$, its radiation damping in both air and water can be computed using Eq. (25) and the Leppington method [in Eq. (27)] for radiation efficiency in the frequency range between 100 and $\overline{10}, 000 \mathrm{~Hz}$, which are compared in Fig. 2. Because the radiation damping is included in the governing equation of the structural bending power flow [22], the difference in the radiation damping could have a large effect on the governing equation of the structural vibration. For example, the radiation damping of the aluminum plate is ranging $0.002-0.0025$ in water, which is about $20-25 \%$ of its original hysteresis damping of the bending motion. However, the radiation damping in air is less than 0.00025 , which is very small compared to the original damping of the structural bending motion, and thus its contribution will be much smaller than that in water.

In addition to the radiation damping, the existence of the dense fluid could affect the power transfer coefficients between the structural panels significantly. The effective structural mass density $\rho_{s}$, the bending wave number $k_{s B}$, and the bending group speed $c_{g B}$ for the structural plates in dense fluid, which are characterized by parameter $\alpha$ in Eq. (26), are included in the analytical method

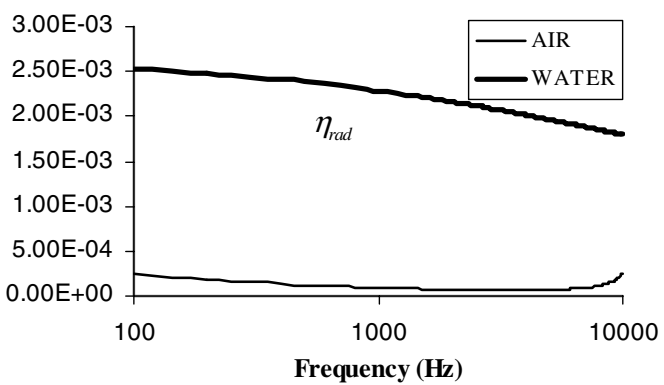

Fig. 2 Radiation damping of an aluminum plate in air and water.

proposed by Langley and Heron [21] to compute power transfer coefficients for structural plates in dense fluid [22]. Figure 3 compares the power transfer coefficients between two identical plates with the same material properties $(E=209 \mathrm{GPa}, v=0.3$, $\rho_{s}=7800 \mathrm{~kg} / \mathrm{m}^{3}$ ) in vacuum, light fluid (air), and dense fluid (water) at frequency $f=2000 \mathrm{~Hz}$. The two plates have the same dimension of $1 \times 1 \times 0.001 \mathrm{~m}^{3}$ and the power transfer coefficients are computed and compared for different junction angles from 0 to $180^{\circ}$. In Fig. $3, \tau_{12}^{B B}$ represents the ratio of energy transfer from the bending energy in plate 1 to the bending energy in plate 2 , and $\tau_{11}^{B B}$ stands for the ratio of energy transfer from the bending energy in plate 1 to the bending energy in plate 1 itself. It is shown that the light fluid (air) does not cause any difference in the power transfer properties compared to those in vacuum. On the contrary, the effect of the dense fluid (water) on the power transfer coefficients is clear, because the power transfer coefficients around $10-30^{\circ}$ and $140^{\circ}$ to $170^{\circ}$ are significantly different in water compared with those in vacuum and air. Thus, the contribution of fluid effect on highfrequency power flow between structural components should be considered.

Once the structural energy solution is available, a conversion matrix $\boldsymbol{W}$ between the nodal bending energy density $\boldsymbol{E}_{s B}$ on the structure and the acoustic power $\boldsymbol{P}$ radiated on each structural element can be established as

$$
\boldsymbol{P}=\boldsymbol{W} \boldsymbol{E}_{s B}
$$

where the component of the conversion matrix is calculated by

$$
W_{i j}=\int_{S_{i}} \delta_{i j} \eta_{\mathrm{rad}}^{i} \omega h \mathrm{~d} S
$$

with $\delta_{i j}=1$ when node $j$ is located on the $i$ th boundary element, and $\delta_{i j}=0$ when node $j$ is not on the $i$ th boundary element.

Combining Eq. (19) with Eq. (28), the governing equation of EBEM can be set up as

$$
\boldsymbol{H} \boldsymbol{\sigma}=\boldsymbol{W} \boldsymbol{E}_{s B}
$$

or in continuum form as

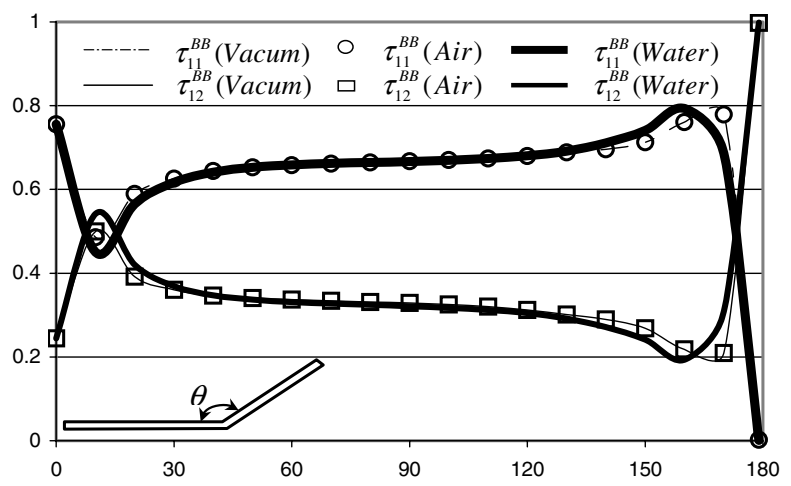

Fig. 3 Power transfer coefficients of two angled plates in vacuum, air, and water. 


$$
h(\boldsymbol{x} ; \boldsymbol{\sigma})=w\left(\boldsymbol{x} ; \boldsymbol{e}_{s B}\right)
$$

where $h(\bullet)$ and $w(\bullet)$ are linear integral forms in terms of the acoustic energy strength and the structural bending energy density, respectively. The solution of Eqs. (30) or (31) then leads to the acoustic source strength for each element on the surface. Therefore, Eqs. (10) and (11) can be readily applied to compute the timeaveraged energy density and energy intensity, respectively, for an arbitrary field point in the free field. Finally, the time-averaged acoustic energy density and energy intensity can be expressed in discrete forms, respectively, as

$$
\begin{aligned}
& e\left(x_{0}\right)=S^{T} \boldsymbol{\sigma} \\
& \boldsymbol{I}\left(\boldsymbol{x}_{0}\right)=\boldsymbol{M} \boldsymbol{\sigma}
\end{aligned}
$$

and the components of vector $\boldsymbol{S}$ and matrix $\boldsymbol{M}$ are derived from Eqs. (10) and (11) as

$$
\begin{gathered}
S_{i}=\frac{1}{A_{i}} \int_{S_{i}} G\left(\boldsymbol{y}, \boldsymbol{x}_{0}\right) \mathrm{d} S \\
M_{i j}=\frac{1}{A_{i}} \int_{S_{i}} H\left(\boldsymbol{y}, \boldsymbol{x}_{0}\right) u_{j}\left(\boldsymbol{y}, \boldsymbol{x}_{0}\right) \mathrm{d} S \quad(j=1,2,3)
\end{gathered}
$$

It is shown that the EFEM-EBEM constitutes a sequential numerical procedure, where the output of EFEM is used to compute the boundary condition for the EBEM. The EBEM is divided into two steps: the first step is to construct the boundary condition using the results from EFEM and solve the acoustic energy strength on the surface; the second step is to calculate the acoustic energy density and acoustic energy intensity in the far-field point in the infinite acoustic domain.

\section{Design Sensitivity Analysis}

\section{A. Direct Differentiation Method}

Consider a given structural-acoustic performance measure, which can be written in an integral form in terms of the time-averaged acoustic energy density $e$ and acoustic energy intensity $\boldsymbol{I}$ in the farfield point as the following:

$$
\psi=\int_{\Omega} g(\boldsymbol{u}, e, \nabla e, \boldsymbol{I}, \nabla \boldsymbol{I}) \mathrm{d} \Omega
$$

The first-order variation of $\psi$ with respect to the design variable $\boldsymbol{u}$ can be obtained as

$$
\psi^{\prime}=\int_{\Omega}\left[g_{, u} \delta \boldsymbol{u}+g_{, e} e^{\prime}+g_{, \nabla e} \cdot \nabla e^{\prime}+g_{, \boldsymbol{I}} \cdot \boldsymbol{I}^{\prime}+g_{, \nabla \boldsymbol{I}}: \nabla \boldsymbol{I}^{\prime}\right] \mathrm{d} \Omega
$$

where the first-order variation of the energy density $e$ with respect to the design $\boldsymbol{u}$ and in the direction of $\delta \boldsymbol{u}$ is defined as

$$
e^{\prime}=e^{\prime}(\boldsymbol{x} ; \boldsymbol{u}, \delta \boldsymbol{u})=\left.\frac{\mathrm{d}}{\mathrm{d} \varepsilon} e(\boldsymbol{x} ; \boldsymbol{u}+\varepsilon \delta \boldsymbol{u})\right|_{\varepsilon=0}=\frac{\partial e^{T}}{\partial \boldsymbol{u}} \delta \boldsymbol{u}
$$

For the structural EFEM, the first-order variation of the structural energy density $\boldsymbol{e}_{s}$ can be obtained directly by differentiating the variational Eq. (2) with respect to the design variables to obtain the sensitivity equation as

$$
\begin{aligned}
& a_{u}\left(\boldsymbol{e}_{s}^{\prime}, \overline{\boldsymbol{e}}_{s}\right)+b_{u}\left(\boldsymbol{e}_{s}^{\prime}, \overline{\boldsymbol{e}}_{s}\right)=\ell_{\delta u}^{\prime}\left(\overline{\boldsymbol{e}}_{s}\right)-a_{\delta u}^{\prime}\left(\boldsymbol{e}_{s}, \overline{\boldsymbol{e}}_{s}\right)-b_{\delta u}^{\prime}\left(\boldsymbol{e}_{s}, \overline{\boldsymbol{e}}_{s}\right) \\
& \quad \forall \overline{\boldsymbol{e}}_{s} \in Z_{s}
\end{aligned}
$$

where the first-order variation of the energy bilinear form $a_{u}(\bullet, \bullet)$, coupling term $b_{u}(\bullet, \bullet)$, and the load linear form $\ell_{\boldsymbol{u}}(\bullet)$ are provided in detail in Choi et al. [40,41].
Because the integral forms $s(\bullet)$ and $\boldsymbol{m}(\bullet)$ in Eqs. (12) and (13) do not explicitly depend on the structural design, the design sensitivity of the energy density and energy intensity at the exterior field point can be obtained by directly differentiating Eqs. (12) and (13), respectively, as

$$
\begin{gathered}
e^{\prime}\left(\boldsymbol{x}_{0}\right)=s\left(\boldsymbol{x}_{0} ; \boldsymbol{\sigma}^{\prime}\right) \\
\boldsymbol{I}^{\prime}\left(\boldsymbol{x}_{0}\right)=\boldsymbol{m}\left(\boldsymbol{x}_{0} ; \boldsymbol{\sigma}^{\prime}\right)
\end{gathered}
$$

where $\sigma^{\prime}$ is the derivative of the acoustic energy strength on the structural boundary elements, which can be obtained by differentiating the governing equation. (31) of EBEM as

$$
\boldsymbol{\sigma}^{\prime}=h^{-1} \cdot w\left(\boldsymbol{x} ; \boldsymbol{e}_{s B}^{\prime}\right)+h^{-1} \cdot w_{\delta \boldsymbol{u}}^{\prime}\left(\boldsymbol{x} ; \boldsymbol{e}_{s B}\right)
$$

where $w_{\delta \boldsymbol{u}}^{\prime}(\square)$ is the first-order variation of the integral form $w(\bullet)$ with respect to the design variable $\boldsymbol{u}$. For the structural damping design variable, it can be seen that, in Eq. (29), $w_{\delta \boldsymbol{u}}^{\prime}(\square)$ vanishes, whereas for the structural thickness design variable, $w_{\delta \boldsymbol{u}}^{\prime}(\square)$ is

$$
w_{\delta \boldsymbol{u}}^{\prime}=\int_{S}\left(\eta_{\mathrm{rad}} \omega \delta h+\omega h \delta \eta_{\mathrm{rad}}\right) \mathrm{d} S
$$

and the expression of $\delta \eta_{\text {rad }}$ is derived by Choi et al. [40,41].

By substituting Eqs. (40-43) into Eq. (37), the sensitivity of the performance measure can be obtained as

$$
\begin{aligned}
\psi^{\prime} & =\int_{\Omega}\left[g_{, \boldsymbol{u}} \delta \boldsymbol{u}+g_{, e} s\left(h^{-1} \cdot w\left(\boldsymbol{e}_{s B}^{\prime}\right)+h^{-1} \cdot w_{\delta \boldsymbol{u}}^{\prime}\left(\boldsymbol{e}_{s B}\right)\right)\right. \\
& +g_{, \nabla e} \cdot \nabla s\left(h^{-1} \cdot w\left(\boldsymbol{e}_{s B}^{\prime}\right)+h^{-1} \cdot w_{\delta \boldsymbol{u}}^{\prime}\left(\boldsymbol{e}_{s B}\right)\right) \\
& +g_{, \boldsymbol{I}} \cdot \boldsymbol{m}\left(h^{-1} \cdot w\left(\boldsymbol{e}_{s B}^{\prime}\right)+h^{-1} \cdot w_{\delta \boldsymbol{u}}^{\prime}\left(\boldsymbol{e}_{s B}\right)\right) \\
& \left.+g_{, \nabla \boldsymbol{I}}: \nabla \boldsymbol{m}\left(h^{-1} \cdot w\left(\boldsymbol{e}_{s B}^{\prime}\right)+h^{-1} \cdot w_{\delta \boldsymbol{u}}^{\prime}\left(\boldsymbol{e}_{s B}\right)\right)\right] \mathrm{d} \Omega
\end{aligned}
$$

Equation (44) provides the expression to calculate the design sensitivity of the performance measure in terms of the design sensitivity of the structural energy density for the direct differentiation method. The solution $\boldsymbol{e}_{s B}^{\prime}$ of the EFEM sensitivity equation (39) then can be substituted into the above equation to get the design sensitivity information of the general structural-acoustic performance measure in Eq. (36).

\section{B. Adjoint Variable Method}

Although the direct differentiation method calculates the sensitivity information through the sensitivity of the structural energy density from EFEM, the adjoint variable method can achieve the same goal by defining an adjoint equation with the adjoint load obtained from the right side of Eq. (44) that is implicitly dependent on the design variables,

$$
\begin{aligned}
& a_{u}\left(\overline{\boldsymbol{\lambda}}_{s}, \boldsymbol{\lambda}_{s}\right)+b_{\boldsymbol{u}}\left(\overline{\boldsymbol{\lambda}}_{s}, \boldsymbol{\lambda}_{s}\right)=\int_{\Omega}\left[g_{, e} s\left(h^{-1} \cdot w\left(\overline{\boldsymbol{\lambda}}_{s}\right)\right)\right. \\
& \quad+g_{, \nabla e} \cdot \nabla s\left(h^{-1} \cdot w\left(\overline{\boldsymbol{\lambda}}_{s}\right)\right)+g_{, \boldsymbol{I}} \cdot \boldsymbol{m}\left(h^{-1} \cdot w\left(\overline{\boldsymbol{\lambda}}_{s}\right)\right) \\
& \left.\quad+g_{, \nabla \boldsymbol{I}}: \nabla \boldsymbol{m}\left(h^{-1} \cdot w\left(\overline{\boldsymbol{\lambda}}_{s}\right)\right)\right] \mathrm{d} \Omega, \quad \forall \overline{\boldsymbol{\lambda}}_{s} \in Z_{s}
\end{aligned}
$$

Evaluation of the above equation at $\bar{\lambda}_{s}=\boldsymbol{e}_{s}^{\prime} \in Z_{s}$ yields

$$
\begin{aligned}
& a_{u}\left(\boldsymbol{e}_{s}^{\prime}, \boldsymbol{\lambda}_{s}\right)+b_{u}\left(\boldsymbol{e}_{s}^{\prime}, \boldsymbol{\lambda}_{s}\right)=\int_{\Omega}\left[g_{, e} s\left(h^{-1} \cdot w\left(\boldsymbol{e}_{s}^{\prime}\right)\right)\right. \\
& \quad+g_{, \nabla e} \cdot \nabla s\left(h^{-1} \cdot w\left(\boldsymbol{e}_{s}^{\prime}\right)\right)+g_{, \boldsymbol{I}} \cdot \boldsymbol{m}\left(h^{-1} \cdot w\left(\boldsymbol{e}_{s}^{\prime}\right)\right) \\
& \left.\quad+g_{, \nabla \boldsymbol{I}}: \nabla \boldsymbol{m}\left(h^{-1} \cdot w\left(\boldsymbol{e}_{s}^{\prime}\right)\right)\right] \mathrm{d} \Omega
\end{aligned}
$$

Similarly, because Eq. (39) holds for arbitrary $\overline{\boldsymbol{e}}_{s} \in Z_{s}$, it can be evaluated at $\overline{\boldsymbol{e}}_{s}=\lambda_{s}$ to obtain

$$
a_{u}\left(\boldsymbol{e}_{s}^{\prime}, \lambda_{s}\right)+b_{u}\left(\boldsymbol{e}_{s}^{\prime}, \lambda_{s}\right)=\ell_{\delta u}^{\prime}\left(\boldsymbol{\lambda}_{s}\right)-a_{\delta u}^{\prime}\left(\boldsymbol{e}_{s}, \lambda_{s}\right)-b_{\delta u}^{\prime}\left(\boldsymbol{e}_{s}, \lambda_{s}\right)
$$


Because Eqs. (46) and (47) have the same left sides, equating their right sides yields

$$
\begin{aligned}
\int_{\Omega} & {\left[g_{, e} s\left(h^{-1} \cdot w\left(\boldsymbol{e}_{s}^{\prime}\right)\right)+g_{, \nabla e} \cdot \nabla s\left(h^{-1} \cdot w\left(\boldsymbol{e}_{s}^{\prime}\right)\right)\right.} \\
& \left.+g_{, \boldsymbol{I}} \cdot \boldsymbol{m}\left(h^{-1} \cdot w\left(\boldsymbol{e}_{s}^{\prime}\right)\right)+g_{, \nabla \boldsymbol{I}}: \nabla \boldsymbol{m}\left(h^{-1} \cdot w\left(\boldsymbol{e}_{s}^{\prime}\right)\right)\right] \mathrm{d} \Omega \\
& =\ell_{\delta \boldsymbol{u}}^{\prime}\left(\boldsymbol{\lambda}_{s}\right)-a_{\delta \boldsymbol{u}}^{\prime}\left(\boldsymbol{e}_{s}, \boldsymbol{\lambda}_{s}\right)-b_{\delta \boldsymbol{u}}^{\prime}\left(\boldsymbol{e}_{s}, \boldsymbol{\lambda}_{s}\right)
\end{aligned}
$$

Because the sensitivity of the performance measure needs the sensitivity of the structural bending energy density, it can be extended to the total structural energy density by simply using a Boolean operation for all the integral forms. As a result, the sensitivity in Eq. (44) can be rewritten as

$$
\begin{aligned}
\psi^{\prime} & =\int_{\Omega}\left[g_{, \boldsymbol{u}} \delta \boldsymbol{u}+g_{, e} s\left(h^{-1} \cdot w\left(\boldsymbol{e}_{s}^{\prime}\right)+h^{-1} \cdot w_{\delta \boldsymbol{u}}^{\prime}\left(\boldsymbol{e}_{s}\right)\right)\right. \\
& +g_{, \nabla e} \cdot \nabla s\left(h^{-1} \cdot w\left(\boldsymbol{e}_{s}^{\prime}\right)+h^{-1} \cdot w_{\delta \boldsymbol{u}}^{\prime}\left(\boldsymbol{e}_{s}\right)\right) \\
& +g_{, \boldsymbol{I}} \cdot \boldsymbol{m}\left(h^{-1} \cdot w\left(\boldsymbol{e}_{s}^{\prime}\right)+h^{-1} \cdot w_{\delta \boldsymbol{u}}^{\prime}\left(\boldsymbol{e}_{s}\right)\right) \\
& \left.+g_{, \nabla \boldsymbol{I}}: \nabla \boldsymbol{m}\left(h^{-1} \cdot w\left(\boldsymbol{e}_{s}^{\prime}\right)+h^{-1} \cdot w_{\delta \boldsymbol{u}}^{\prime}\left(\boldsymbol{e}_{s}\right)\right)\right] \mathrm{d} \Omega
\end{aligned}
$$

where the definitions of the integral forms $h(\bullet), w(\bullet), s(\bullet)$, and $\boldsymbol{m}(\bullet)$ are all extended from structural bending energy density to the total structural energy density variables. Substitution of Eq. (48) into Eq. (49) yields

$$
\begin{aligned}
\psi^{\prime} & =\int_{\Omega}\left[g_{, \boldsymbol{u}} \delta \boldsymbol{u}+g_{, e} s\left(h^{-1} \cdot w_{\delta \boldsymbol{u}}^{\prime}\left(\boldsymbol{e}_{s}\right)\right)+g_{, \nabla e} \cdot \nabla s\left(h^{-1} \cdot w_{\delta \boldsymbol{u}}^{\prime}\left(\boldsymbol{e}_{s}\right)\right)\right. \\
& \left.+g_{, \boldsymbol{I}} \cdot \boldsymbol{m}\left(h^{-1} \cdot w_{\delta \boldsymbol{u}}^{\prime}\left(\boldsymbol{e}_{s}\right)\right)+g_{, \nabla \boldsymbol{I}}: \nabla \boldsymbol{m}\left(h^{-1} \cdot w_{\delta \boldsymbol{u}}^{\prime}\left(\boldsymbol{e}_{s}\right)\right)\right] \mathrm{d} \Omega \\
& +\ell_{\delta \boldsymbol{u}}^{\prime}\left(\boldsymbol{\lambda}_{s}\right)-a_{\delta \boldsymbol{u}}^{\prime}\left(\boldsymbol{e}_{s}, \boldsymbol{\lambda}_{s}\right)-b_{\delta \boldsymbol{u}}^{\prime}\left(\boldsymbol{e}_{s}, \boldsymbol{\lambda}_{s}\right)
\end{aligned}
$$

In the above equation, the explicitly dependent terms from EFEM such as $\ell_{\delta \boldsymbol{u}}^{\prime}(\square, \square), a_{\delta \boldsymbol{u}}^{\prime}(\square, \square)$, and $b_{\delta \boldsymbol{u}}^{\prime}(\square, \square)$ have been discussed extensively by Choi et al. [40,41] for analytical design components, such as structural plates.

Because the conversion integral $w(\bullet)$ in Eq. (31) is dependent of the structural design parameters, the calculation of the variation of the conversion integral $w_{\delta u}^{\prime}(\square)$ will only involve the structural EFEM model. Thus, the above sensitivity equation is shown to be an integration on the structural part only, even though the adjoint load computation will require the information of the acoustic EBEM model information.

In a typical structural-acoustic analysis as will be shown in the following examples, the number of performance measures will be much less than the number of design variables, where the adjoint variable method will have a big advantage over the finite difference method in solving the number of matrix equations. For example, in a passenger car design, if the performance is measured as the acoustic pressure at the driver's ear position, but the design variables are chosen as the material properties and thickness of all structural panels, the adjoint variable method just needs to solve Eq. (45) once and the sensitivity will only involve the integration of Eq. (50) for each design variable. But the finite difference method will need to solve Eqs. (2), (12), and (13) for each design variable 2 or 3 times to get the sensitivity results.

\section{Numerical Implementation}

\section{A. Direct Differentiation Method}

After applying the Gauss quadrature to all integral forms, the matrix equation to solve for the elemental acoustic energy strength is obtained from Eq. (30) as

$$
\boldsymbol{\sigma}=\boldsymbol{H}^{-1} \boldsymbol{W} \boldsymbol{E}_{s B}
$$

and the time-averaged acoustic energy density and acoustic energy intensity in the far-field point $\boldsymbol{x}_{0}$ can be obtained using Eqs. (32) and (33), respectively.
The first-order variation of the elemental acoustic energy strength can be obtained by discretizing Eq. (42) as

$$
\boldsymbol{\sigma}^{\prime}=\boldsymbol{H}^{-1} \boldsymbol{W} \boldsymbol{E}_{s B}^{\prime}+\boldsymbol{H}^{-1} \boldsymbol{W}^{\prime} \boldsymbol{E}_{s B}
$$

where the first-order variation of the structural bending energy density vector $\boldsymbol{E}_{s B}^{\prime}$ can be obtained by solving the discretized form of Eq. (39),

$$
\boldsymbol{K}_{s}\left(+\boldsymbol{J}_{\mathrm{ss}}\right) \boldsymbol{E}_{s}^{\prime}=\boldsymbol{F}^{\mathrm{fic}}
$$

The calculation of the fictitious load vector $\boldsymbol{F}^{\text {fic }}$ can be found in Choi et al. $[40,41]$ for the parametric design variables. Finally, the variation of the acoustic energy density and acoustic energy intensity can be calculated by discretized forms of Eqs. (40) and (1ㅡ) as

$$
\begin{gathered}
e^{\prime}\left(\boldsymbol{x}_{0}\right)=\boldsymbol{S}^{T} \boldsymbol{\sigma}^{\prime} \\
\boldsymbol{I}^{\prime}\left(\boldsymbol{x}_{0}\right)=\boldsymbol{M} \boldsymbol{\sigma}^{\prime}
\end{gathered}
$$

Substitution of Eqs. (54) and (55) into Eq. (37) and implementing numerical integration yields the design sensitivity of the performance measure.

\section{B. Adjoint Variable Method}

Because the computation of the adjoint load vector depends on the system matrix of both structural EFEM and acoustic EBEM, two steps need to be carried out to get the adjoint load vector. First, four separate acoustic adjoint load vectors are defined on an acoustic adjoint reanalysis as

$$
\boldsymbol{H}^{T} \boldsymbol{\xi}=\boldsymbol{S}
$$

$$
\boldsymbol{H}^{T} \boldsymbol{\zeta}_{i}=\boldsymbol{M}_{i} \quad(i=1,2,3)
$$

where the acoustic adjoint loads are obtained from the acoustic geometry and the location of the exterior field point $\boldsymbol{x}_{0}$. Vector $\boldsymbol{M}_{i}$ is the $i$ th column vector of the matrix $\boldsymbol{M}$. Once the acoustic adjoint responses $\xi$ and $\zeta=\left\{\zeta_{1}, \zeta_{2}, \zeta_{3}\right\}^{T}$ are solved from an acoustic reanalysis, the intermediate adjoint load vectors are defined on the product of the acoustic adjoint responses with the conversion matrix $\boldsymbol{W}$ as

$$
\begin{aligned}
& \boldsymbol{F}_{e}=\boldsymbol{W}^{T} \boldsymbol{\xi} \\
& \boldsymbol{F}_{\boldsymbol{I}}=\boldsymbol{W}^{T} \boldsymbol{\zeta}
\end{aligned}
$$

where $\boldsymbol{F}_{e}$ is the intermediate adjoint load contributed from the acoustic energy density and $\boldsymbol{F}_{\boldsymbol{I}}$ is the intermediate adjoint load contributed from the acoustic energy intensity at the exterior field point.

By substituting the intermediate adjoint load vectors into the right side of the adjoint equation (45), the discrete structural adjoint load is obtained as

$$
\boldsymbol{F}_{\text {adj }}=g_{, e} \boldsymbol{F}_{e}+g_{, \nabla e} \cdot \nabla\left(\boldsymbol{F}_{e}\right)+g_{, \boldsymbol{I}} \cdot\left(\boldsymbol{F}_{\boldsymbol{I}}\right)+g_{, \nabla \boldsymbol{I}}: \nabla\left(\boldsymbol{F}_{\boldsymbol{I}}\right)
$$

The adjoint response can be discretized using the finite element shape functions as

$$
\lambda_{s}=\sum_{i=1}^{N E} N_{s}^{i} \lambda_{s}^{i}=\boldsymbol{N}_{s}^{T} \boldsymbol{\Lambda}_{s}
$$

Then the adjoint equation (45) can be discretized as

$$
\left(\boldsymbol{K}_{s}+\boldsymbol{J}_{\mathrm{ss}}\right)^{T} \boldsymbol{\Lambda}_{s}=\boldsymbol{F}_{\text {adj }}
$$

After solving the structural adjoint response $\boldsymbol{\Lambda}_{s}$ at the nodal points, the finite element approximation of the variations of the structural energy bilinear form, structural-structural coupling term, and load 
linear form, and the variation of the conversion matrix in Eqs. (29) and (43) can be applied to derive the variation of the performance measure in the discrete form as

$$
\begin{aligned}
\psi^{\prime} & =\iint_{\Omega} g_{, \boldsymbol{u}} \cdot \delta \boldsymbol{u} \mathrm{d} \Omega+\boldsymbol{\Lambda}_{s}^{T} \boldsymbol{F}^{\mathrm{fic}}+g_{, e} \boldsymbol{\xi}^{T} \boldsymbol{W}^{\prime} \boldsymbol{E}_{s}+g_{, \nabla e} \cdot \nabla\left(\boldsymbol{\xi}^{T} \boldsymbol{W}^{\prime} \boldsymbol{E}_{s}\right) \\
& +g_{, \boldsymbol{I}} \cdot\left(\boldsymbol{\zeta}^{T} \boldsymbol{W}^{\prime} \boldsymbol{E}_{s}\right)+g_{, \nabla \boldsymbol{I}}: \nabla\left(\boldsymbol{\zeta}^{T} \boldsymbol{W}^{\prime} \boldsymbol{E}_{s}\right)
\end{aligned}
$$

From the definition of the acoustic adjoint load vectors in Eqs. (56) and (57), it can be seen that as long as the geometry of the boundary element model holds fixed, the acoustic adjoint load vectors and thus the acoustic adjoint responses remain unchanged. This provides great advantages for the parametric design optimization of the structural-acoustic model, because as long as the structural geometry does not change, the parametric design variables such as panel thickness and material damping will not influence the computation of the acoustic loads and acoustic adjoint responses. However, the structural adjoint loads are dependent on structural designs through the conversion matrix $\boldsymbol{W}$. As a result, Eqs. (58-62) need to be evaluated at each design iteration in the sizing design optimization process.

\section{Numerical Examples}

\section{A. Design Sensitivity Analysis of Radiation of the Simplified Van} Model in Air

The radiated noise from the vibrating structure to the exterior domain is as important as the interior noise because it generates noise pollution. The exterior noise radiated from a vibrating vehicle structure is one of the most common radiation problems to study. To design vehicle structure such that the radiated noise level is controlled under a regulatory limit is an important task for noise, vibration, and harshness (NVH) design. A practical application of EFEM-EBEM is to design a vehicle structure with optimized NVH performance and ride quality at high frequency.

To test the proposed NVH DSA method for high-frequency problems, a simplified passenger vehicle model is constructed and studied (Fig. 4). The corresponding EFEM model (Fig. 5) is composed of $\overline{118}$ structural plate elements, which are integrated through 56 structural-structural joints. An analytical method developed by Langley and Heron [21] is used to compute the power transfer coefficients between plate members, and the radiation efficiency method is used to compute the power transfer coefficients between plate members and acoustic domain.

The passenger vehicle model is composed of seven different structural panels that are made of aluminum. Those panels have the material property of Young's modulus $E=71 \mathrm{GPa}$, Poisson's ratio $\nu=0.33$, and mass density $\rho_{s}=2700 \mathrm{~kg} / \mathrm{m}^{3}$. All the panels have the same thickness $h=10 \mathrm{~mm}$ and the hysteresis damping factor of $\eta_{s}=0.01$ is used for each panel. The noise is radiated into acoustic space filled with air that has mass density $\rho_{0}=1.02 \mathrm{~kg} / \mathrm{m}^{3}$, hysteresis damping $\eta_{a}=0.001$, and wave speed $c_{0}=343 \mathrm{~m} / \mathrm{s}$.

A structural EFEM analysis is performed first without considering the interior acoustic domain to obtain the structural energy density

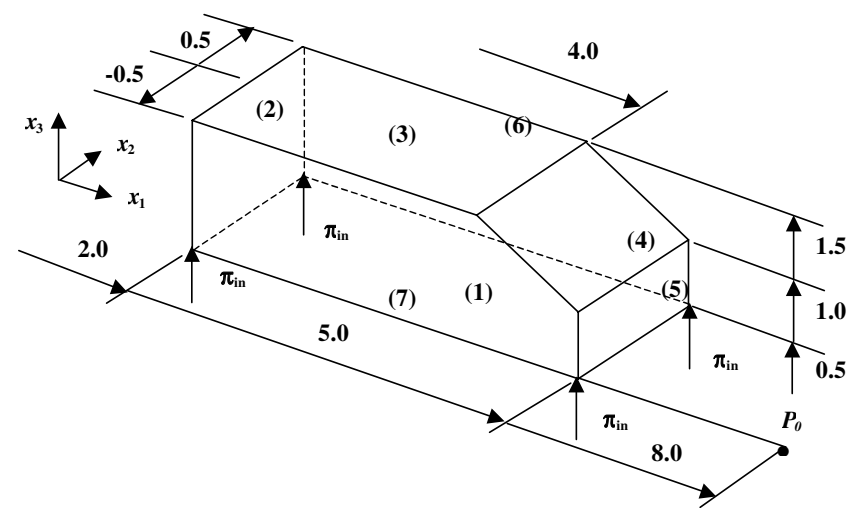

Fig. 4 Recovery point in exterior domain of simplified van model.

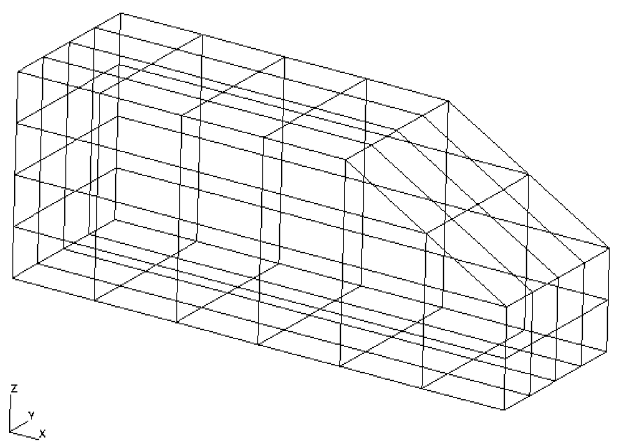

Fig. 5 Energy finite element model of simplified van model.

distribution. Because the structural model does not have any inner part, the acoustic energy boundary element model is the same as the structural finite element model in Fig. $\underline{5}$. A recovery point $P_{0}$ is chosen to be located at the left front of the vehicle with a coordinate at $(8.0,-0.5,0.5)$ as shown in Fig. 4. The acoustic EBEM analysis, following the structural EFEM analysis, predicts the noise level of $70.1 \mathrm{~dB}$ at the recovery point $P_{0}$. The proposed adjoint design sensitivity method is carried out to study accuracy of the proposed DSA method for the radiated noise. The thickness and damping of each structural panel are selected as design variables and $1 \%$ of the initial design is perturbed where the central finite difference method is used to verify the sensitivity coefficients obtained using the proposed DSA method. Comparisons of the results from the proposed design sensitivity method (shown in the sixth column) and central finite difference method (shown in the fifth column) are shown in Table 1 for the thickness and damping design variables. Excellent agreement is observed between these two sets of numerical results, where $100 \%$ in the last column indicates that these two results are the same, which proves the accuracy of the proposed DSA method.

The sensitivity information shown in Table 1 indicates different effects of the structural designs on the noise level at the recovery point $P_{0}$. All the damping designs have negative contributions to the radiated noise, and most of the thickness designs also have negative contributions, except panels 2 and 3, which have positive sensitivity coefficients, which means increasing thicknesses in panels 2 and 3 will increase the radiated noise at $P_{0}$.

\section{B. Design Sensitivity Analysis of Underwater Radiation of Undersea Vehicle Model}

The high-frequency noise radiation also plays an important role in naval structures as in automotive and aerospace structures. For example, reducing the noise radiated from a vibrating undersea ship to make it avoid being identified is an important topic in the naval structural design. A small undersea vehicle model is constructed and the noise radiation in water is analyzed. The vehicle model is $6.0 \mathrm{~m}$ long, and the maximum inner diameter is $0.522 \mathrm{~m}$. The thickness of the vehicle skin is $10 \mathrm{~mm}$, and all four of the bulkheads have the same thickness of $25.4 \mathrm{~mm}$. The energy finite element model in Fig. 6 is composed of 1744 nodes and 1060 elements. There are totally $\overline{5} 90$ structural joints in the model. The structural parts have material properties of Young's modulus $E=70 \mathrm{GPa}$, Poisson's ratio $v=0.3$, mass density $\rho_{s}=2700 \mathrm{~kg} / \mathrm{m}^{3}$, and hysteresis damping factor $\eta_{s}=0.01$. The acoustic medium is water with which the undersea model interacts, and has mass density $\rho_{0}=1000 \mathrm{~kg} / \mathrm{m}^{3}$, wave speed $c_{0}=1500 \mathrm{~m} / \mathrm{s}$, and hysteresis damping factor $\eta_{a}=0.001$. The undersea vehicle model can be divided into 11 substructures as shown in Fig. 6. Axisymmetric excitation is applied in the middle of substructure 5 .

While the energy finite element model consists of both inner and outer parts, the energy boundary element model only consists of the outer parts of the structural model (Fig. 7). The structural energy density is converted to the power flow on each structural element, which is then used as the boundary condition to solve for the acoustic energy strength for each boundary element. The radiated noise at an 
Table 1 Design sensitivity analysis results for exterior noise radiation from the simplified van model

\begin{tabular}{lccccrr}
\hline \hline \multirow{2}{*}{ Design variables } & \multicolumn{2}{c}{ Performance measure } & & FDM & AVM & Ratio \\
\cline { 3 - 4 } & & $\psi(\boldsymbol{u}-\delta \boldsymbol{u})$ & $\psi(\boldsymbol{u}+\delta \boldsymbol{u})$ & $\delta \psi / \delta \boldsymbol{u}$ & \multicolumn{1}{c}{$\psi^{\prime}$} & $\%$ \\
\hline \multirow{2}{*}{ Panel 1 } & Thickness $h_{1}$ & 70.212718 & 70.005966 & $-0.103376 \mathrm{E}+04$ & $-0.103371 \mathrm{E}+04$ & 100.01 \\
& Damping $\eta_{1}$ & 70.122588 & 70.108509 & $-0.703957 \mathrm{E}+03$ & $-0.703956 \mathrm{E}+03$ & 100.00 \\
\multirow{2}{*}{ Panel 2 } & Thickness $h_{2}$ & 70.116585 & 70.118256 & $0.835712 \mathrm{E}+01$ & $0.835866 \mathrm{E}+01$ & 99.98 \\
& Damping $\eta_{2}$ & 70.117710 & 70.113976 & $-0.186710 \mathrm{E}+03$ & $-0.186710 \mathrm{E}+03$ & 100.00 \\
\multirow{2}{*}{ Panel 3 } & Thickness $h_{3}$ & 70.116369 & 70.117665 & $0.648013 \mathrm{E}+01$ & $0.648394 \mathrm{E}+01$ & 99.94 \\
& Damping $\eta_{3}$ & 70.119146 & 70.111944 & $-0.360090 \mathrm{E}+03$ & $-0.360090 \mathrm{E}+03$ & 100.00 \\
\multirow{2}{*}{ Panel 4 } & Thickness $h_{4}$ & 70.119748 & 70.109103 & $-0.532236 \mathrm{E}+02$ & $-0.532125 \mathrm{E}+02$ & 100.02 \\
& Damping $\eta_{4}$ & 70.117610 & 70.113476 & $-0.206683 \mathrm{E}+03$ & $-0.206683 \mathrm{E}+03$ & 100.00 \\
Panel 5 & Thickness $h_{5}$ & 70.117944 & 70.110138 & $-0.390287 \mathrm{E}+02$ & $-0.390208 \mathrm{E}+02$ & 100.02 \\
& Damping $\eta_{5}$ & 70.116552 & 70.114534 & $-0.100905 \mathrm{E}+03$ & $-0.100905 \mathrm{E}+03$ & 100.00 \\
\multirow{2}{*}{ Panel 6 } & Thickness $h_{6}$ & 70.119946 & 70.114788 & $-0.257887 \mathrm{E}+02$ & $-0.257789 \mathrm{E}+02$ & 100.04 \\
& Damping $\eta_{6}$ & 70.120740 & 70.110355 & $-0.519246 \mathrm{E}+03$ & $-0.519245 \mathrm{E}+03$ & 100.00 \\
Panel 7 & Thickness $h_{7}$ & 70.121715 & 70.104112 & $-0.880149 \mathrm{E}+02$ & $-0.880005 \mathrm{E}+02$ & 100.02 \\
& Damping $\eta_{7}$ & 70.120835 & 70.110259 & $-0.528774 \mathrm{E}+03$ & $-0.528774 \mathrm{E}+03$ & 100.00 \\
\hline \hline
\end{tabular}

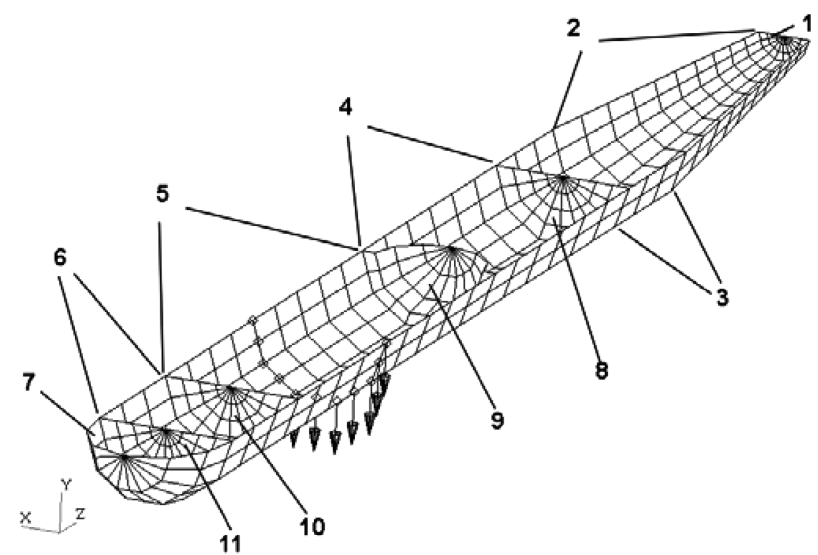

Fig. 6 Energy finite element model and substructures of a small undersea vehicle.

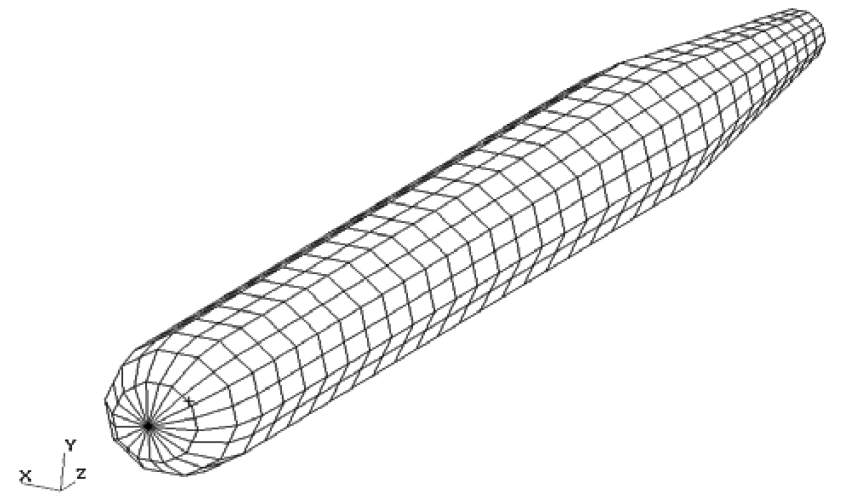

Fig. 7 Energy boundary element model of a small undersea vehicle model. arbitrary exterior point can be computed by using Green's function. To predict the exterior radiation noise caused by the vibrating vehicle model, a series of points at distance $100 \mathrm{~m}$ from the center of the vehicle model is selected as recovery points as shown in Fig. 8. Because the model and excitation are axisymmetric, all the points on the circle in Fig. 8 will have the same noise level. Therefore, the noise level on the entire circle can be represented by one point, for example, point $P_{0}$.

Furthermore, if each substructure of the vehicle model can be considered as a design component, the design sensitivity of the noise level on the entire circle will also be equivalent to the design sensitivity of the noise level at point $P_{0}$. The radiation analysis using EFEM-EBEM predicted the noise level at the circle to be $118.87 \mathrm{~dB}$ when the excitation frequency is $f=2000 \mathrm{~Hz}$. Following the DSA procedure discussed in the previous sections, the sensitivity coefficients of the noise level with respect to the thickness and damping factor of each substructure are calculated and summarized in Table 2. Without exception, all the damping factors have negative sensitivity coefficients. For the thickness design, thicknesses of substructures 1-7 have positive sensitivities. Among the four bulkheads, substructure 9 has positive sensitivity while the other three have negative sensitivities. Thus at the current design, increasing damping of all structural panels and decreasing thickness of substructures 1-7 and 9, while increasing thickness of other substructures, is the desirable way to reduce the radiated noise at the points of interest.

\section{Multi-Objective Design Optimization of Underwater Noise Reduction}

In this section, a design optimization problem is formulated and solved for the undersea vehicle model. The DSA formulation derived in the previous sections is integrated into the design optimization program to carry out design optimization.

The design optimization process is illustrated in Fig. 9. The EFEM is used to compute the structural energy distribution and power radiated from the structural plate, which is used as the boundary conditions for EBEM to predict the noise radiation at far-field points. Using the sequential adjoint variable method, DSA calculates the

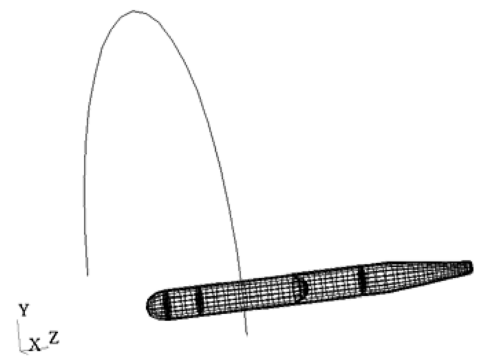

a) View 1

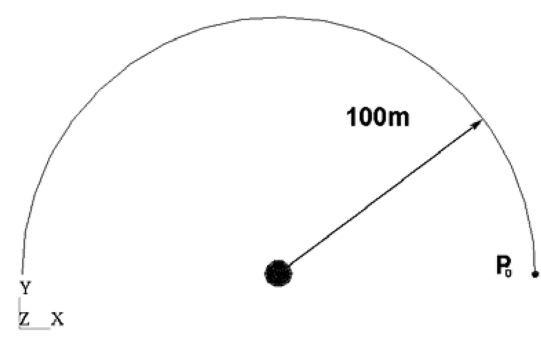

b) View 2

Fig. 8 Exterior noise recovery position of a small undersea vehicle model. 
Table 2 Design sensitivity analysis results for exterior noise radiation from a small undersea vehicle model

\begin{tabular}{|c|c|c|c|c|c|c|}
\hline \multirow[t]{2}{*}{ Design variables } & & \multicolumn{2}{|c|}{ Performance measure } & \multirow{2}{*}{$\begin{array}{l}\text { FDM } \\
\delta \psi / \delta u\end{array}$} & \multirow{2}{*}{$\begin{array}{c}\text { AVM } \\
\psi^{\prime}\end{array}$} & \multirow{2}{*}{$\begin{array}{c}\text { Ratio } \\
\%\end{array}$} \\
\hline & & $\psi(u-\delta u)$ & $\psi(u+\delta u)$ & & & \\
\hline \multirow{2}{*}{ Substructure } & & 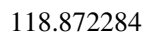 & 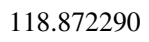 & 0.3 & 0.3 & 100 \\
\hline & & & & 1 & & U. \\
\hline \multirow{2}{*}{ Substructure 2} & & 04 & 98 & 6997 & +01 & 0.01 \\
\hline & & & & +01 & - & 00.00 \\
\hline \multirow{2}{*}{ Substructure 3} & & & & & & 00.00 \\
\hline & & & & 1 & & 00.00 \\
\hline \multirow{2}{*}{ Substructure } & & & & & & 0.00 \\
\hline & & & & 2 & -0 & 0.00 \\
\hline \multirow{2}{*}{ Substructure 5} & & & & & 3 & 00.00 \\
\hline & & & & 3 & -0 & 100.00 \\
\hline \multirow{2}{*}{ Substructure 6} & & 118.8 & 118. & $0.815108 \mathrm{E}+01$ & $0.815076 \mathrm{E}+01$ & 100.00 \\
\hline & & & & $-0.725600 \mathrm{E}+01$ & $-0.725598 \mathrm{E}+01$ & 100.00 \\
\hline \multirow{2}{*}{ Substructure } & & & & & & 100.00 \\
\hline & & & & & & 00.00 \\
\hline \multirow{2}{*}{ Substructure 8} & & & & -0.2 & -0.2 & 100.00 \\
\hline & & & & -0.2207 & -0.2 & 100.00 \\
\hline \multirow{2}{*}{ Substructure 9} & & & & & 0.4 & 100.00 \\
\hline & & & & $-0.149853 \mathrm{E}$ & -0.14985 & 100.00 \\
\hline \multirow{2}{*}{ Substructure 10} & Thickness $h_{10}$ & 118.872409 & 118.872148 & $-0.513201 \mathrm{E}+00$ & $-0.513172 \mathrm{E}+00$ & 100.00 \\
\hline & & 118. & & $-0.104580 \mathrm{E}+01$ & $-0.104580 \mathrm{E}+01$ & 100.00 \\
\hline \multirow{2}{*}{ Substructure 11} & Thick & 118.872288 & & $-0.188094 \mathrm{E}+00$ & $-0.188102 \mathrm{E}+00$ & 100.00 \\
\hline & $\operatorname{lng} \eta_{11}$ & 118.872319 & 118.872241 & $-0.387373 \mathrm{E}+00$ & $-0.387372 \mathrm{E}+00$ & 100.00 \\
\hline
\end{tabular}

derivatives of the far-field radiation noise with respect to the design variables, which are used by the design optimization algorithm (such as SLP-sequential linear programming and SQP-sequential quadratic programming, etc.) to search for the optimum design.

The objective of the design optimization is to reduce the noise level at far-field points for the high-frequency range between 1600 and $2500 \mathrm{~Hz}$ while minimizing the vehicle mass. The acoustic performance measure is the far-field noise level at the point $P_{0}$. On the other hand, higher energy density on the structure could increase the structural stress level. The computational results show that the highest energy density occurs on the excitation circle. One of the nodes on the circle, node 752 , is used as the structural performance measure. The acoustic performance measure at $P_{0}$ and the structural performance measure at node 752 are obtained at the center frequencies of the $1 / 3$ octave bands in the range between 1600 and $2500 \mathrm{~Hz}$ as shown in Table 3 .

The vehicle mass is the design objective, whereas the above noise level and structural energy density will serve as the design constraints. In Table 3 , the maximum noise level occurs at $f=1600 \mathrm{~Hz}$, which is $118.92 \mathrm{~dB}$ with reference value of $2.0 \mathrm{E}-5 \mathrm{~Pa}$, so the first constraint is set to reduce the highest pressure level to $116.00 \mathrm{~dB}$ with reference value of $1.0 \mathrm{E}-12 \mathrm{~J} / \mathrm{m}^{2}$, which means $2.9 \mathrm{~dB}$ reduction, which is equivalent to $28.4 \%$ noise reduction. At the same time, the highest structural energy density occurs at $f=1600 \mathrm{~Hz}$, which is $126.02 \mathrm{~dB}$, so the second constraint is to keep the highest structural energy density level under $126.0 \mathrm{~dB}$.

The vehicle mass depends on the thickness design variables and is expressed by

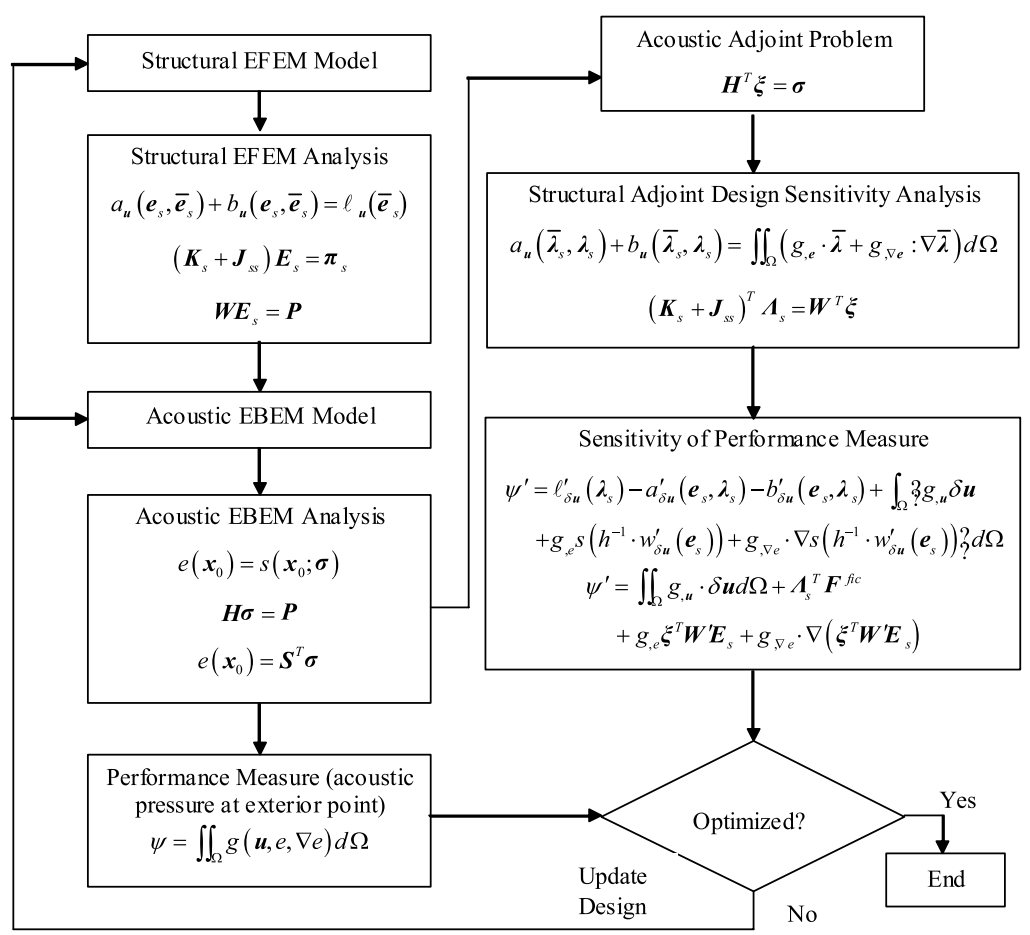

Fig. 9 Design optimization procedure for a high-frequency radiation problem using the energy finite element method and the energy boundary element method. 
Table 3 Performance measures at initial design

\begin{tabular}{lcc}
\hline \hline $\begin{array}{l}\text { Frequency } \\
\mathrm{Hz}\end{array}$ & $\begin{array}{r}\text { Acoustic pressure } \\
\text { dB, RE2.0E-5 }\end{array}$ & $\begin{array}{c}\text { Structural energy density } \\
\text { dB, RE1.0E-12 }\end{array}$ \\
\hline 1600 & 118.92 & 126.02 \\
1692 & 118.91 & 125.84 \\
1789 & 118.90 & 125.58 \\
1891 & 118.89 & 125.37 \\
2000 & 118.87 & 125.17 \\
2115 & 118.86 & 125.06 \\
2236 & 118.84 & 124.79 \\
2364 & 118.83 & 124.52 \\
2500 & 118.82 & 124.32 \\
\hline \hline
\end{tabular}

$$
f_{1}=\sum_{\mathrm{NE}} \int_{\Omega_{i}} \rho_{i} h_{i} \mathrm{~d} \Omega
$$

where NE is the total number of the finite elements, $\rho_{i}$ the mass density of the $i$ th finite element, and $h_{i}$ is the thickness of the $i$ th finite element.

The vehicle mass associated with the damping design variables is determined according to the damping adjustment: free layer damping or constrained layer damping treatment [44]. The former is also called surface damping treatment in which a "high-loss" material (usually rubber, asphalt, vinyl) is sprayed on, brushed on, or adhesively bonded to the panel surface as shown in Fig. 10a. The latter is a treatment where a relatively thin layer of damping material is sandwiched between the structure and a stiff but thin cover plate as a retaining layer, such as aluminum, steel, or sheet metal (Fig. 10b). Although the constrained layer damping treatment has better damping enhancement than the free layer damping, the free layer damping is much easier and more practical in engineering applications. For example, the asphalt sheets are widely used in automotive, naval, and aerospace industry. On the other hand, the constrained layer damping needs an additional aluminum layer for the damping treatment, and it needs to be extruded, slit, and cut into preferred shapes, which requires more material and labor cost involved than the free layer damping treatment.

Some research has been carried out in the prediction of the mechanical properties of different surface damping treatments, such as the RKU analysis developed by Ross, Ungar, and Kerwin [45]. It is shown that the properties of the laminated material are functions of the thickness and material properties of each layer. However, the damping materials are usually provided in standard products from manufacturers, whose thicknesses are not adjustable. Moreover, the material properties of the mostly used damping materials, including the mass density, Young's modulus, Poisson's ratio, and hysteresis damping factor are unknown or largely variant in most cases. As a result, it is difficult to analytically model the damping mass directly related to the total vehicle mass. However, the mass contributed by the damping adjustment is proportional to the amount of damping adjustment and the area on which the adjustment is applied. To simplify the problem in this study, the mass of the damping material adjustment is assumed to be linear in terms of the damping increment and the surface area as

$$
f_{2}=\sum_{\mathrm{NE}} \int_{\Omega_{i}}\left(\eta_{i}-\eta_{i}^{\text {ori }}\right) \mathrm{d} \Omega
$$

where $\eta_{i}^{\text {ori }}$ is the original damping on the $i$ th finite element, and $\eta_{i}$ is the proposed damping computed by the design optimization algorithm.

Because of the existence of two different objective functions in Eqs. (64) and (65), a multi-objective design optimization needs to be carried out for this problem. In this paper, the multi-objective design optimization problem is transformed into a single-objective design optimization problem, where the weighted objective method is used to convert the multi-objective functions into a single-objective function by a weighted sum as

$$
f=w_{1} f_{1}+w_{2} f_{2}
$$

where $w_{1}$ and $w_{2}$ are weighting coefficients, with the following relationship:

$$
w_{1}+w_{2}=1
$$

The optimization results of Eq. (66) are infinitely many, subject to the different combination of weighting coefficients. If the coefficients are selected at a discrete set of points, a corresponding discrete set of optimization results will be obtained, that is, the Pareto optimal set. The design engineers can pick the values of the results from the Pareto optimal set, based on different design preferences. As a result, the design optimization problem is formulated as follows:

Objective: Minimize the weighted objective function

$$
f=w_{1} \frac{f_{1}}{F_{1}}+w_{2} \frac{f_{2}}{F_{2}}
$$

where $0.5<f_{1} / F_{1}<1.5, \quad 0<f_{2} / F_{2}<1, \quad 0<w_{1}<1, \quad$ and $0<w_{2}<1$.

Constraints: Subject to two sets of constraints:

1) reduce noise level at far-field point $P_{0}$ in $1600-2500 \mathrm{~Hz}$ to $116.0 \mathrm{~dB}$;

2) keep the highest structural energy density level in 1600 $2500 \mathrm{~Hz}$ below $126.0 \mathrm{~dB}$.

Parametric design variables:

11 panel thickness ( $\pm 50 \%$ design change allowed);

11 panel damping ( $+200 \%$ design change allowed), where in Eq. (68), the objective functions $f_{1}$ and $f_{2}$ are normalized by the maximum allowable mass and damping cost changes $F_{1}$ and $F_{2}$, which are distances between the upper and lower limits of the thickness and damping design space.

The design optimization is carried out for the different combinations of weighting coefficients $w_{1}$ and $w_{2}$ as shown in Table 4, and DOT (design optimization tool) [46] is used as the optimization tool where SQP is chosen as the optimization algorithm. The Pareto optimal results are obtained and listed in Table 4, while the Pareto optimal set is plotted in Fig. 11, which shows that the weighted objective function is convex, and the reduction of one objective function needs to compromise the other.

To illustrate the optimization results, the history of the vehicle mass cost for the case when $w_{1}=1$ and $w_{2}=0$ is plotted in Fig. 12, from which we can see that the optimization is converged after four iterations, and at the end of the optimization, the vehicle mass is reduced from 306 to $172 \mathrm{Kg}$, a $134 \mathrm{Kg}$ reduction. Such a large amount of mass reduction is achieved by maximizing the use of the damping material, where all the damping design variables increase to the maximum allowable value (Table 5). Compared with the damping design variables, all the thickness design variables decrease to the minimum allowable value to reduce the mass, except the thickness of substructure 5 , which reduces by a certain amount but
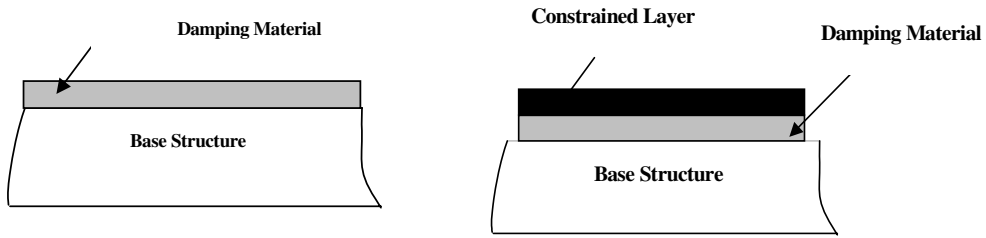

a) Free layer damping

b) Constrained layer damping

Fig. 10 Damping treatments. 
Pareto Optimum Set

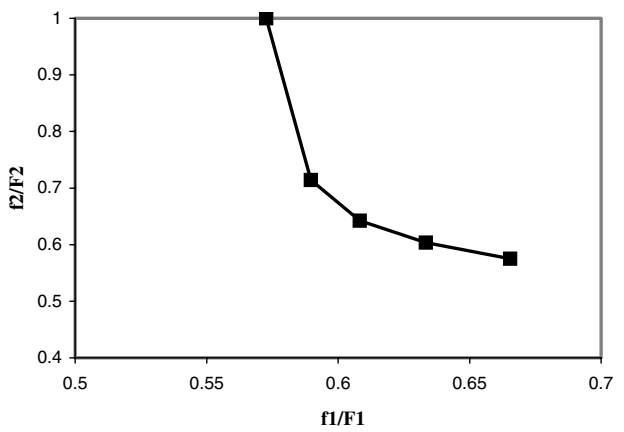

Fig. 11 Pareto optimal set of multi-objective optimization.

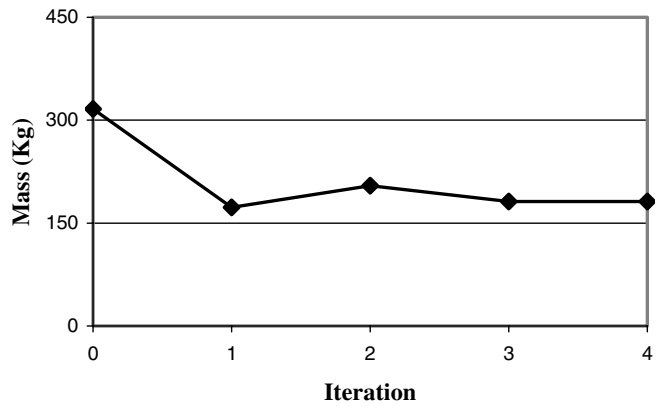

Fig. 12 History of vehicle mass cost function.

does not hit the lower boundary of the design space to keep the design constraints satisfied.

The optimum design results of the thickness and damping design variables are listed in Table $\underline{5}$, which provides design engineers

Table 4 Pareto optimal results for different weighting coefficients

\begin{tabular}{lccccc}
\hline \hline$w_{1}$ & 1.0 & 0.75 & 0.5 & 0.25 & 0.0 \\
$w_{2}$ & 0.0 & 0.25 & 0.5 & 0.75 & 1.0 \\
$f_{1} / F_{1}$ & 0.573 & 0.590 & 0.608 & 0.633 & 0.665 \\
$f_{1}, \mathrm{Kg}$ & 181.3 & 186.5 & 192.4 & 200.3 & 210.5 \\
$f_{2} / F_{2}$ & 1.000 & 0.714 & 0.642 & 0.604 & 0.575 \\
\hline \hline
\end{tabular}

guidance for design modification under different requirements. If the contribution of damping material to vehicle mass is ignored, then all the damping design variables are increased to the upper bound and the thickness design variables are reduced. Once the contribution of damping material to the vehicle mass is considered in the total vehicle mass with increased weighting coefficient $w_{2}$, the damping design variables need to be decreased to reduce the mass, while the thickness design variables need to be increased to meet the design constraints. If the contribution of the metal thickness to vehicle mass is ignored, most of the damping design variables are reduced to the lower bound except the damping in substructures 5 and 6 , which stays at the upper bound to satisfy the design constraints. On the contrary, all thicknesses decrease to the lower bound, except that the thicknesses of substructures 8 and 11 increase to the upper bound and the thickness of substructure 5 decreases to a certain level above the lower bound to help satisfy the design constraints.

\section{Conclusions}

A continuum DSA of high-frequency radiation problems using a sequential EFEM-EBEM procedure is formulated and presented. The direct differentiation method calculates the sensitivity of the exterior noise through the sensitivity of the structural energy density obtained from EFEM. The adjoint variable method calculates the adjoint response of a structural EFEM reanalysis under the adjoint load obtained from an acoustic EBEM reanalysis. For the adjoint variable method, a sequential adjoint analysis process is developed. The sensitivity information is obtained by carrying out numerical integration only on the structural FE part. It is successfully applied in the design of automotive and naval structures to search for the best material layout to achieve lowest noise level at high frequency. The DSA method presents excellent numerical accuracy compared with the finite difference results in the numerical examples of the noise radiation of automotive and naval structures in light and dense fluids, and the DSA information has been used in a multi-objective optimization of naval structure to achieve the optimum design where the structural mass is minimized and the high-frequency underwater noise radiation is reduced.

\section{Acknowledgement}

This research is supported by the Automotive Research Center sponsored by the U.S. Army Tank Automotive Research, Development and Engineering Center (TARDEC) under contract

Table 5 Thickness and damping design results for Pareto optimal set

\begin{tabular}{|c|c|c|c|c|c|c|}
\hline & Original design & $\begin{array}{l}w_{1}=1.0 \\
w_{2}=0.0\end{array}$ & $\begin{array}{l}w_{1}=0.75 \\
w_{2}=0.25\end{array}$ & $\begin{array}{c}w_{1}=0.5 \\
w_{2}=0.5\end{array}$ & $\begin{array}{l}w_{1}=0.25 \\
w_{2}=0.75\end{array}$ & $\begin{array}{l}w_{1}=0.0 \\
w_{2}=1.0\end{array}$ \\
\hline$h_{1}$ & $1.00 \mathrm{E}-02$ & $5.00 \mathrm{E}-03$ & $5.00 \mathrm{E}-03$ & $5.00 \mathrm{E}-03$ & $5.00 \mathrm{E}-03$ & $5.00 \mathrm{E}-03$ \\
\hline$h_{2}$ & $1.00 \mathrm{E}-02$ & $5.00 \mathrm{E}-03$ & $5.00 \mathrm{E}-03$ & $5.00 \mathrm{E}-03$ & $5.00 \mathrm{E}-03$ & $5.00 \mathrm{E}-03$ \\
\hline$h_{3}$ & $1.00 \mathrm{E}-02$ & $5.00 \mathrm{E}-03$ & $5.00 \mathrm{E}-03$ & $5.00 \mathrm{E}-03$ & $5.00 \mathrm{E}-03$ & $5.00 \mathrm{E}-03$ \\
\hline$h_{4}$ & $1.00 \mathrm{E}-02$ & $5.00 \mathrm{E}-03$ & $5.00 \mathrm{E}-03$ & $5.00 \mathrm{E}-03$ & $5.00 \mathrm{E}-03$ & $5.00 \mathrm{E}-03$ \\
\hline$h_{5}$ & $1.00 \mathrm{E}-02$ & $7.85 \mathrm{E}-03$ & 7.93E-03 & $7.88 \mathrm{E}-03$ & 7.87E-03 & $7.89 \mathrm{E}-03$ \\
\hline$h_{6}$ & $1.00 \mathrm{E}-02$ & $5.00 \mathrm{E}-03$ & $5.00 \mathrm{E}-03$ & $5.00 \mathrm{E}-03$ & $5.00 \mathrm{E}-03$ & $5.00 \mathrm{E}-03$ \\
\hline$h_{7}$ & $1.00 \mathrm{E}-02$ & $5.00 \mathrm{E}-03$ & $5.00 \mathrm{E}-03$ & $5.00 \mathrm{E}-03$ & $5.00 \mathrm{E}-03$ & $5.00 \mathrm{E}-03$ \\
\hline$h_{8}$ & $2.54 \mathrm{E}-02$ & $1.27 \mathrm{E}-02$ & 2.09E-02 & $3.21 \mathrm{E}-02$ & $2.93 \mathrm{E}-02$ & $3.81 \mathrm{E}-02$ \\
\hline$h_{9}$ & $2.54 \mathrm{E}-02$ & $1.27 \mathrm{E}-02$ & $1.27 \mathrm{E}-02$ & $1.27 \mathrm{E}-02$ & $1.27 \mathrm{E}-02$ & $1.27 \mathrm{E}-02$ \\
\hline$h_{10}$ & $2.54 \mathrm{E}-02$ & $1.27 \mathrm{E}-02$ & $1.27 \mathrm{E}-02$ & $1.27 \mathrm{E}-02$ & $1.27 \mathrm{E}-02$ & $1.27 \mathrm{E}-02$ \\
\hline$h_{11}$ & $2.54 \mathrm{E}-02$ & $1.27 \mathrm{E}-02$ & $1.27 \mathrm{E}-02$ & $1.27 \mathrm{E}-02$ & $2.88 \mathrm{E}-02$ & $3.81 \mathrm{E}-02$ \\
\hline$\eta_{1}$ & $1.00 \mathrm{E}-02$ & $3.00 \mathrm{E}-02$ & $1.46 \mathrm{E}-02$ & $1.02 \mathrm{E}-02$ & $1.00 \mathrm{E}-02$ & $1.00 \mathrm{E}-02$ \\
\hline$\eta_{2}$ & $1.00 \mathrm{E}-02$ & $3.00 \mathrm{E}-02$ & $1.80 \mathrm{E}-02$ & $1.02 \mathrm{E}-02$ & $1.00 \mathrm{E}-02$ & $1.00 \mathrm{E}-02$ \\
\hline$\eta_{3}$ & $1.00 \mathrm{E}-02$ & $3.00 \mathrm{E}-02$ & $1.95 \mathrm{E}-02$ & $1.03 \mathrm{E}-02$ & $1.00 \mathrm{E}-02$ & $1.00 \mathrm{E}-02$ \\
\hline$\eta_{4}$ & $1.00 \mathrm{E}-02$ & $3.00 \mathrm{E}-02$ & $2.04 \mathrm{E}-02$ & $2.44 \mathrm{E}-02$ & $2.39 \mathrm{E}-02$ & $2.64 \mathrm{E}-02$ \\
\hline$\eta_{5}$ & $1.00 \mathrm{E}-02$ & $3.00 \mathrm{E}-02$ & $3.00 \mathrm{E}-02$ & $3.00 \mathrm{E}-02$ & $3.00 \mathrm{E}-02$ & $3.00 \mathrm{E}-02$ \\
\hline$\eta_{6}$ & $1.00 \mathrm{E}-02$ & $3.00 \mathrm{E}-02$ & $3.00 \mathrm{E}-02$ & $3.00 \mathrm{E}-02$ & $3.00 \mathrm{E}-02$ & $3.00 \mathrm{E}-02$ \\
\hline$\eta_{7}$ & $1.00 \mathrm{E}-02$ & $3.00 \mathrm{E}-02$ & $3.00 \mathrm{E}-02$ & $2.78 \mathrm{E}-02$ & $1.65 \mathrm{E}-02$ & $1.16 \mathrm{E}-02$ \\
\hline$\eta_{8}$ & $1.00 \mathrm{E}-02$ & $3.00 \mathrm{E}-02$ & $1.00 \mathrm{E}-02$ & $1.00 \mathrm{E}-02$ & $1.00 \mathrm{E}-02$ & $1.00 \mathrm{E}-02$ \\
\hline$\eta_{9}$ & $1.00 \mathrm{E}-02$ & $3.00 \mathrm{E}-02$ & $3.00 \mathrm{E}-02$ & $3.00 \mathrm{E}-02$ & $3.00 \mathrm{E}-02$ & $1.06 \mathrm{E}-02$ \\
\hline$\eta_{10}$ & $1.00 \mathrm{E}-02$ & $3.00 \mathrm{E}-02$ & $3.00 \mathrm{E}-02$ & $3.00 \mathrm{E}-02$ & $3.00 \mathrm{E}-02$ & $2.32 \mathrm{E}-02$ \\
\hline$\eta_{11}$ & $1.00 \mathrm{E}-02$ & $3.00 \mathrm{E}-02$ & $1.00 \mathrm{E}-02$ & $1.00 \mathrm{E}-02$ & $1.00 \mathrm{E}-02$ & $1.00 \mathrm{E}-02$ \\
\hline
\end{tabular}


DAAE07-94-C-R094. The authors gratefully acknowledge this support.

\section{References}

[1] Zienkiewicz, O. C., and Taylor, R. L., The Finite Element Method: Basic Formulation and Linear Problems, 4th ed., McGraw-Hill Book Co., Ltd., London, England, 1989.

[2] Hughes, T. J. R., The Finite Element Method, Prentice-Hall, Englewood Cliffs, NJ, 1987.

[3] Bathe, K. J., Finite Element Procedures, Prentice-Hall, Englewood Cliffs, NJ, 1996.

[4] Brebbia, C. A., and Dominguez, J., Boundary Elements An Introductory Course, Computational Mechanics Publications, Boston, MA, 1989.

[5] Banerjee, P. K., and Butterfield, R., Boundary Element Methods in Engineering Science, McGraw-Hill, New York, 1981.

[6] Kythe, P. K., Introduction to Boundary Element Methods, CRC Press, Boca Raton, FL, 1995.

[7] Atalla, N., and Bernhard, R. J., "Review of Numerical Solutions for Low-Frequency Structural-Acoustic Problems," Applied Acoustics, Vol. 43, No. 3, 1994, pp. 271-294.

[8] Lyon, R., and Eichler, E., "Random Vibration of Connected Structures," Journal of Acoustic Society of America, Vol. 36, No. 7, July 1964, pp. 1344-1354.

[9] Lyon, R., Statistical Energy Analysis of Dynamical Systems: Theory and Application, MIT Press, Cambridge, MA, 1975.

[10] Lyon, R., and DeJong, R. G., Theory and Application of Statistical Energy Analysis, 2nd ed., Butterworth-Heinemann, Boston, MA, 1995.

[11] Nefske, D. J., Wolf, J. A., and Howell, L. J., "Structural-Acoustic Finite Element Analysis of the Automobile Passenger Compartment: A Review of Current Practice," Journal of Sound and Vibration, Vol. 80, No. 2, 1982, pp. 247-266.

[12] Wohlever, J., and Bernhard, R. J., "Mechanical Energy Flow Models of Rods and Beams," Journal of Sound and Vibration, Vol. 153, No. 1, 1992, pp. 1-19.

[13] Bouthier, O. M., and Bernhard, R. J., "Models of Space-Averaged Energetics of Plates," AIAA Journal, Vol. 30, No. 3, 1992, pp. 616-623.

[14] Bouthier, O. M., "Energetics of Vibrating Systems," Ph.D. Thesis, Purdue University, West Lafayette, IN, 1992.

[15] Bouthier, O. M., and Bernhard, R. J., "Simple-Models of the Energetics of Transversely Vibrating Plates," Journal of Sound and Vibration, Vol. 182, No. 1, 1995, pp. 149-166.

[16] Cho, P. E., and Bernhard, R. J., "Energy Flow Analysis of Coupled Beams," Journal of Sound and Vibration, Vol. 211, No. 4, 1998, pp. 593-605.

[17] Cho, P. E., "Energy Flow Analysis of Coupled Structures," Ph.D. Thesis, Purdue University, West Lafayette, IN, 1993.

[18] Bernhard, R. J., and Huff, J. E., "Structural-Acoustic Design at High Frequency Using the Energy Finite Element Method," Journal of Vibration and Acoustics, Vol. 121, No. 3, 1999, pp. 295-301.

[19] Zhang, W., Wang, A., and Vlahopoulos, N., "An Alternative Energy Finite Element Formulation Based on Incoherent Orthogonal Waves and Its Validation for Marine Structures," Finite Elements in Analysis and Design, Vol. 38, No. 12, Oct. 2002, pp. 1095-1113.

[20] Bitsie, F., "The Structural-Acoustic Energy Finite Element Method and Energy Boundary Element Method," Ph.D. Thesis, Purdue University, West Lafayette, IN, 1996.

[21] Langley, R. S., and Heron, K. H., "Elastic Wave Transmission Through Plate/Beam Junctions," Journal of Sound and Vibration, Vol. 143, No. 2, 1990, pp. 241-253.

[22] Zhang, W., Wang, A., and Vlahopoulos, N., "High Frequency Vibration Analysis of Thin Elastic Plates Under Heavy Fluid Loading by an Energy Finite Element Formulation," Journal of Sound and Vibration, Vol. 263, No. 1, 2003, pp. 21-46.

[23] Vlahopoulos, N., "A Numerical Structure-Borne Noise Prediction Scheme Based on the Boundary-Element Method, with a New Formulation for the Singular-Integrals," Computers and Structures, Vol. 50, No. 1, 1994, pp. 97-109.

[24] Zhang, W., Wang, A., and Vlahopoulos, N., "Validation of the EFEA Method Through Correlation with Conventional FEA and SEA Results," SAE Paper 2001-01-1618, 2001.

[25] Wang, S., "Theory and Applications of a Simplified Energy Finite Element Method and Its Similarity to SEA,"Noise Control Engineering Journal, Vol. 50, No. 2, March-April 2002, pp. 63-72.

[26] Wang, A., Vlahopoulos, N., and Wu, K., "Development of An Energy Boundary Element Formulation of Sound Radiation at High
Frequency," Journal of Sound and Vibration, Vol. 278, Nos. 1-2, 2004, pp. 413-436.

[27] Choi, K. K., and Lee, J. H., "Sizing Design Sensitivity Analysis of Dynamic Frequency Response of Vibrating Structures," ASME Journal of Mechanical Design, Vol. 114, No. 1, 1992, pp. 166-173.

[28] Choi, K. K., Shim, I., and Wang, S., "Design Sensitivity Analysis of Structure-Induced Noise and Vibration," Journal of Vibration and Acoustics, Vol. 119, No. 2, 1997, pp. 173-179.

[29] Kim, N. H., Dong, J., Choi, K. K., Vlahopoulos, N., Ma, Z. D., Castanier, M., and Pierre, C., "Design Sensitivity Analysis for Sequential Structural-Acoustic Problems," Journal of Sound and Vibration, Vol. 263, No. 3, 2003, pp. 569-591.

[30] Dong, J., Choi, K. K., and Kim, N. H., "Design Optimization of Structural-Acoustic Problem Using FEA-BEA with Adjoint Variable Method," ASME Journal of Mechanical Design, Vol. 126, No. 3, May 2004, pp. 527-533.

[31] Ma, Z. D., and Hagiwara, I., "Sensitivity Analysis-Method for Coupled Acoustic-Structural Systems Part 1: Modal Sensitivities," AIAA Journal, Vol. 29, No. 1, 1991, pp. 1787-1795.

[32] Ma, Z. D., and Hagiwara, I., "Sensitivity Analysis-Method for Coupled Acoustic-Structural Systems Part 2: Direct Frequency-Response and Its Sensitivities," AIAA Journal, Vol. 29, No. 1, 1991, pp. 1796-1801.

[33] Ma, Z. D., and Hagiwara, I., "Development of Eigenmode and Frequency Response Sensitivity Analysis Methods for Coupled Acoustic-Structural Systems," JSME International Journal, Series III, Vol. 35, No. 2, 1995, pp. 229-235.

[34] Kane, J. H., Mao, S., and Everstine, G. C., "Boundary Element Formulation for Acoustic Shape Sensitivity Analysis," Journal of the Acoustical Society of America, Vol. 90, No. 1, 1991, pp. 561-573.

[35] Cunefare, K. A., and Koopman, G. H., "Acoustic Design Sensitivity for Structural Radiators," Journal of Vibration and Acoustics, Vol. 114, No. 2, 1992, pp. 179-186.

[36] Koo, B. U., "Shape Design Sensitivity Analysis of Acoustic Problems Using a Boundary Element Method," Computers and Structures, Vol. 65, No. 5, 1997, pp. 713-719.

[37] Matsumoto, T., Tanaka, M., and Yamada, Y., "Design Sensitivity Analysis of Steady-State Acoustic Problems Using Boundary Integral Equation Formulation," JSME International Journal Series C, Vol. 38, No. 1, 1995, pp. 9-16.

[38] Smith, D. C., and Bernhard, R. J., "Computation of Acoustic Shape Design Sensitivity Using A Boundary Element Method," Journal of Vibration and Acoustics-Transactions of The ASME, Vol. 114, No. 1, Jan. 1992, pp. 127-132.

[39] Kim, N. H., Dong, J., and Choi, K. K., "Energy Flow Analysis and Design Sensitivity Analysis of Structural-Acoustic Problems at High Frequency," Journal of Sound and Vibration, Vol. 269, Nos. 1-2, 2004, pp. 213-250.

[40] Choi, K. K., Dong, J., Vlahopoulos, N., Wang, A., and Zhang, W., "Parametric Design Sensitivity Analysis of High Frequency StructuralAcoustic Problems Using Energy Finite Element Method," ASME 2003 Design Engineering Technical Conferences and Computers and Information in Engineering Conference, ILDETC/DAC-48753, American Society of Mechanical Engineers, Fairfield, NJ, 2003.

[41] Dong, J., Choi, K. K., Wang, A., Zhang, W., and Vlahopoulos, N., "Parametric Design Sensitivity Analysis of High Frequency StructuralAcoustic Problems Using Energy Finite Element Method," International Journal for Numerical Methods in Engineering, Vol. 62, No. 1, 2005, pp. 83-121.

[42] Cremer, L., Heckl, L., and Ungar, E. E., Structure-Bourn Sound, 2nd ed., Springer-Verlag, Berlin, 1988.

[43] Leppington, F. G., Broadbent, E. G., F. R. S., and Heron, K. H., "The Acoustic Radiation Efficiency of Rectangular Panels," Proceedings of the Royal Society of London A, Vol. 382, No. 1783, 1982, pp. 245-271.

[44] Nashif, A. D., Jones, D. I. G., and Henderson, J. P., Vibration Damping, Wiley, New York, 1985.

[45] Ross, D., Ungar. E. E., and Kerwin, E. M. Jr.,, "Damping of Plate Flexural Vibrations by Means of Viscoelastic Laminates," Structural Damping (papers presented at a colloqium on structural damping held at the ASME annual meeting in Atlantic City, NJ, Dec. 1959), American Society of Mechanical Engineers, New York, 1959, pp. 49-88.

[46] Vanderplaats, G. N., "DOT User's Manual," VMA Corp. , Colorado Springs, CO, 1997. 